\title{
Analytical Methods for Polar Pollutants
}

\author{
Thorsten Reemtsma and José Benito Quintana
}

\section{1}

\section{Introduction}

The last few decades have shown that analytical chemistry and environmental chemistry are "conjoined twins". Neither can move significantly forward without the contribution and support of the other discipline. But in their conjoined development both disciplines have contributed much to our knowledge of environmental pollution, to the understanding of environmental processes, and to the development of measures and strategies to reduce contamination.

Complementary to the following book chapters that focus on the occurrence and behavior of different classes of polar pollutants in the water cycle, this chapter provides an overview of the recent status of the other half of the "conjoined twins", the analytical methods to determine these polar pollutants. This subject could easily be the topic of an independent book. Condensing it to one chapter requires considerable selectivity. Therefore, analytical strategies and approaches to the trace analysis of polar pollutants from environmental samples are outlined rather than described in detail.

\section{2}

\section{The Analytical Process}

A scheme of the analytical process for the determination of polar pollutants in water and particulate samples (sludge, sediment and soil) is presented in Fig. 1.1. This scheme excludes sampling, which is outside of the scope of this overview. Obviously not all the steps presented in this scheme are always necessary, and in many cases, for example, clean-up or derivatization prior to GC determination and sometimes even the enrichment step can be skipped.

The most common and important steps will be considered in separate sections, paying special attention to the most relevant techniques and to expected future developments, according to current trends in analytical chemistry: e.g., miniaturization, automation, reduction in solvent consumption, and sample manipulation. 


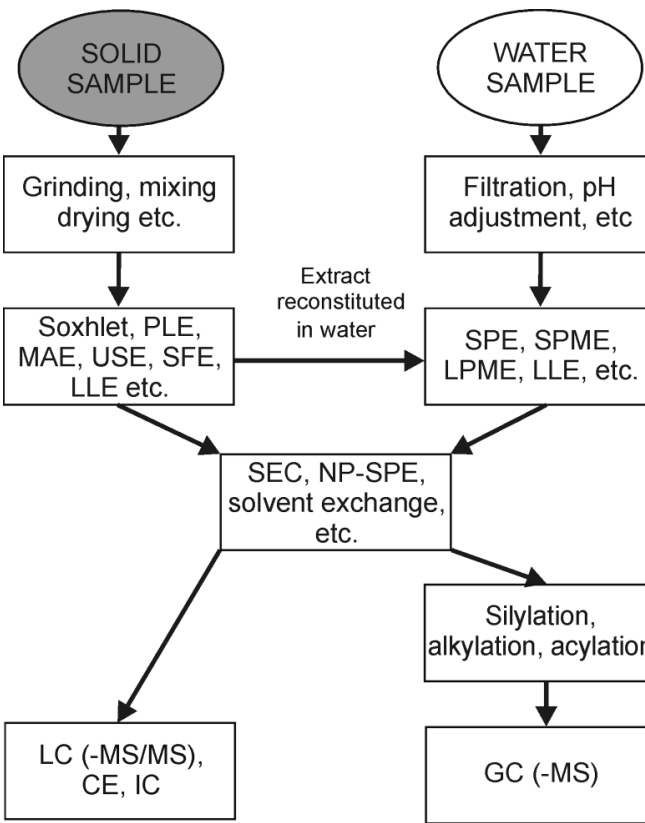

\section{SAMPLE PRETREATMENT}

EXTRACTION \& ENRICHMENT

CLEAN-UP

DERIVATIZATION

SEPARATION \& DETERMINATION
Fig. 1.1 Overview of the analytical process (excluding sampling). CE: Capillary Electrophoresis, IC: Ion Chromatography, GC: Gas Chromatography, LC: Liquid Chromatography, LLE: Liquid Liquid Extraction, LPME: Liquid Phase Microextraction, MAE: Microwave Assisted Extraction, MS: Mass Spectrometry,
NP: Normal Phase, PLE: Pressurized Liquid Extraction, SEC: Size Exclusion Chromatography, SFE: Supercritical Fluid Extraction, SPE: Solid Phase Extraction, SPME: Solid Phase Microextraction, USE: Ultrasound Assisted Extraction.

Examples will be presented from different compound classes that are covered in this book and that exhibit different physicochemical properties. The analytical methods for some of these compound classes, namely surfactants [1, 2], herbicides and other pesticides [3-9], pharmaceuticals [10-13], disinfection byproducts [14], and complexing agents $[15,16]$, have been the subject of specific reviews, which can provide the reader with more detailed information.

1.3

\section{Sample Pretreatment and Analyte Extraction}

\subsection{1}

\section{Sample Pretreatment}

Several steps may be required after sampling and before analyte extraction and final determination. These steps include sample preservation, filtration, $\mathrm{pH}$ adjustment of aqueous samples, drying and homogenization of solid samples, etc. They 
are very straightforward, but if they are not performed properly the original sample composition may be seriously altered by these steps.

Even sample storage and shipping can be a critical step in sample preparation. For instance, significant losses of salicylic acid, acetaminophen, and fenofibrate were observed from a mixture of 12 acidic pharmaceuticals spiked to a treated wastewater that was stored in the dark at $4{ }^{\circ} \mathrm{C}$ [17]. It may be advisable to analyze samples as soon as possible or to store samples in the dark at $-20^{\circ} \mathrm{C}$ if they cannot be analyzed immediately. Sample storage may also influence the relative importance of adducts like sulfates and glucoronides as compared to the parent compound [18].

Another common step for sample preservation is acidification, but analytes may not be stable at certain $\mathrm{pH}$ values. For example, some fibrate drugs hydrolyze to clofibric acid and fenofibric acid at pH 2 [19], and tetracyclines may undergo epimerization [11]. Furthermore, $\mathrm{pH}$ adjustment should be carried out after filtration in order to avoid possible losses during filtration due to the increase in the analyte hydrophobicity.

\subsection{2}

\section{Solid Samples}

To date, the analysis of polar organic contaminants in the water cycle has focused on the aqueous phase, whereas particulate material has not been much considered. Therefore, analytical methods for the determination of polar compounds from solid samples, mainly sediment and sludge, are less developed [20]. Although sorption may not be considered a relevant process for many polar organic compounds, its importance gradually increases with decreasing polarity and increasing solids concentration. Moreover, complexation of ionic and ionizable polar pollutants may occur through inorganic constituents of the matrix, especially in the case of sediments and soils $[8,12]$. Thus, in the development of extraction methods for sorbed compounds, one needs to consider their properties, hydrophobicity, and acid-base properties, as well as those of the particulate phase. To develop appropriate extraction conditions that are able to overcome the matrix-analyte interactions, one needs to know whether these interactions are primarily hydrophobic or electrostatic.

In contrast, methods for the determination of pesticides from soil samples are comparatively well developed [8]. Additionally, reviews have appeared recently on the determination of pharmaceuticals $[11,12]$, surfactants, and their metabolites $[1,2]$ in environmental solid samples.

The classical extraction method, both for polar and non-polar analytes, was Soxhlet extraction, which consumes large amounts of solvent as well as of the sample, and which is relatively time consuming. Therefore, current methods tend to minimize the consumption of solvents, sample amount, and extraction time by providing additional energy and/or pressure to the mixture of sample and solvent. This supports desorption and diffusion of the analytes from the sample to the solution and enhances their solubility in the extraction media. The different methods are distinguished by the way the energy is supplied to the system and the kind of ex- 
tracting fluid employed, namely: microwave assisted extraction (MAE), supercritical fluid extraction (SFE), pressurized liquid extraction (PLE), and ultrasound assisted extraction (USE) [21, 22]. Most of them have been fully automated, which is another major advantage over Soxhlet extraction. For example antibiotics were extracted from agricultural soils by PLE [23] at room temperature and $1000 \mathrm{kPa}$ to avoid tetracycline degradation at high temperatures, but allowing the process to be automated.

A first class of compounds that may be considered is phosphoric acid triesters. They are non-ionic and do not have ionizable groups, so they somewhat resemble classical hydrophobic organic pollutants. However, these compounds cover a broad polarity group, from relatively polar short-chain alkyl phosphates (e.g., triethylphosphate and trichloroethylphosphate; $\log K_{\text {ow }} 0.09$ and 1.43 respectively) to quite hydrophobic long-chain alkyl phosphates and aryl phosphates (e.g., triphenylphosphate; $\log K_{\text {ow }} 4$.76) [24]. Thus, a typical method designed for extracting PAHs or PCBs based on Soxhlet extraction with hexane or toluene works very well for the non-polar analytes but not for the most polar ones, while choosing an intermediate polarity solvent or a solvent mixture provides acceptable recoveries for the whole group of analytes. In one of the pioneering works on MAE, this was compared to Soxhlet extraction and the shake-flask system using a mixture of ethyl acetate and dichloromethane [25]. Microwave extraction yielded better recoveries except for the very polar trimethylphosphate.

More hydrophobic ionizable compounds can also be extracted by an appropriate solvent. The biocides triclosan and triclocarban have been extracted with dichloromethane [26] or acetone/methanol mixtures [27, 28]. Several pharmaceuticals can be extracted also by acetone and methanol from sediment [29], sludge [30], and suspended particulate material [31]. The clean-up by reextraction from water was achieved by using different SPE sorbents and $\mathrm{pH}$ values for the different pharmaceutical classes $[29,30]$.

In the case of more polar and ionizable analytes, however, pure organic solvents are not adequate extractants. Fluoroquinolones are amphoteric species, and their charge state depends on the $\mathrm{pH}$ value. Even when their net charge is zero, they are present in the zwitterionic form. For that reason, best recoveries for PLE of fluoroquinolone antibiotics from sludge and soil were obtained by a mixture of acetonitrile and water (1/1) [32]. Moreover, acidification ( $\mathrm{pH} 2)$ further improved the extraction efficiency, and this was attributed not only to the enhanced solubility of fluoroquinolones at acidic $\mathrm{pH}$ but also to the protonation of the acidic sites of the matrix constituents. Finally, the clean-up of the extracts was accomplished by SPE, employing a mixed-phase cation-exchange disk cartridge, like the method for the extraction of fluoroquinolones from water samples [33]. In a similar way, Crescenzi et al. [34] extracted triazine herbicides from soil by hot water containing a phosphate buffer at $\mathrm{pH} 7.5$ in a fully automated process.

The use of hot (subcritical) water extraction is an innovative way of extracting analytes of different polarity from solid matrices. Though water is a rather polar solvent at $20^{\circ} \mathrm{C}$, its dielectric constant decreases markedly as the temperature is raised to $200^{\circ} \mathrm{C}$, and it is then able to efficiently extract hydrophobic compounds, 
e.g., PAHs [35, 36]. Hence, the polarity of water may be matched to the analyte polarity by selecting an optimized extraction temperature. A good example of this is the extraction of surfactants from sludge. Surfactants comprise a broad group of compounds with different chemical properties, including basic, acidic, and neutral compounds. As a result, most analytical methods are dedicated to one or two compound classes [1]. However the use of subcritical water at $\mathrm{pH} 9.4$ allows the efficient extraction of more than 10 different acidic and neutral chemical groups of surfactants, providing better recoveries than Soxhlet extraction for the nonylphenol ethoxy carboxylates [37]. A clear advantage of using water as a solvent is the ecological aspect and its straightforward application to reverse-phase SPE or SPME clean-up without need for solvent evaporation.

As mentioned previously, the kind of interaction (hydrophilic or hydrophobic) between the analyte and the matrix constituents is another critical point in the extraction. In the case of ionic interactions, the $\mathrm{pH}$ of the extraction solution may be shifted or chemicals may be added that can compete with the analytes for the matrix constituents. This technique is used in the case of phenoxyacid herbicide extraction from soils and sediments, where the addition of EDTA to the extracting solvent has been proven to improve recoveries [38-40]. The proposed mechanisms of the simultaneous extraction and derivatization of 2,4-D from soil by PLE are represented in Fig. 1.2 [39]. The same is true for the extraction of tetracycline antibiotics, where a buffer containing EDTA or an acid with chelating properties (e.g., citric acid) is employed to overcome the complexation of these analytes with sample cations [12, 23].

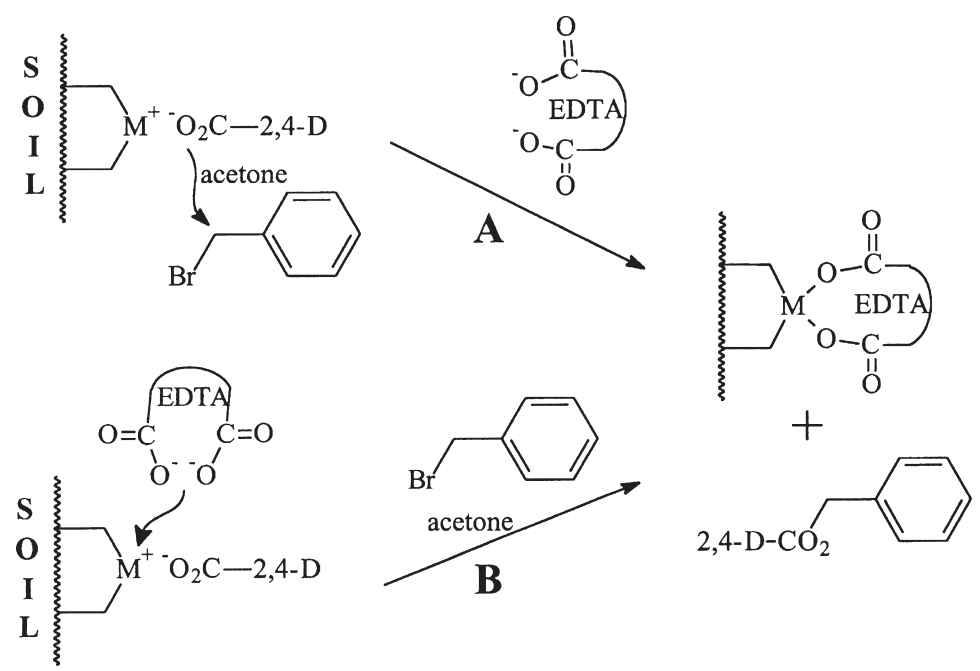

Fig. 1.2 Suggested mechanisms of the PLE-PFBBr derivatization of the herbicide 2, 4-D from soil ( $F$ atoms not represented): (a) 2,4-D is released while being derivatized with PFBBr, then EDTA occupies its position at the soil surface (b) EDTA replaces 2,4-D from the active surface site, then the freely dissolved EDTA is derivatized by PFBBr. Reprinted from [39], with permission from Elsevier. 
After extraction, the resulting extracts from solid samples, particularly in the case of sludge, normally need to be purified before analysis. This has been done in most cases by SPE of the extracts, either employing normal-phase materials (silica, florisil, etc.) if the analytes are relatively non-polar [26, 31, 41, 42] or by reverse and ion exchange phase sorbents if the analytes are relatively polar or possess ionic groups [23, 27, 29, 30, 32]. In many cases a method developed for the SPE of water samples was employed for this purpose after reconstituting or diluting the extract with water.

\subsection{3}

\section{Aqueous Samples}

The determination of polar contaminants in water samples is normally preceded by an extraction step in order to enrich the analytes of interest. This extraction should be as selective as possible in order to minimize the coextraction of matrix that may interfere with analyte detection.

Several extraction techniques for aqueous samples are available, with SPE being the standard procedure. LLE has remained important for only a few applications, e.g., the determination of haloacetic acids [14]. In fact, the US-EPA has two methods available for their determination: one based on SPE [43] and the other based on LLE [44], where, however, the volume of extracting solvent has been minimized to $4 \mathrm{~mL}$ of MTBE. The alternatives to SPE are microextraction techniques, namely SPME and, more recently, LPME, as they consume less organic solvent or sample volume (or virtually none in the case of SPME) [45]. Both SPE and microextractions are discussed in more detail.

Other techniques used for the analysis of volatile compounds, like headspace (HS) and purge and trap (PT), are applicable to very few of the polar target analytes considered here (e.g., some haloacetic acids [46]) because of the often ionic character and high water solubility of many polar compounds.

\subsubsection{Solid Phase Extraction}

As already mentioned, SPE is nowadays the most widely used extraction technique for polar organic analytes in water samples. SPE is very convenient; it can be automated and adapted to various analytes by a proper selection from the wide range of sorbent materials available. In the case of polar analytes, the breaking point has been the development of new polystyrene-divinylbenzene (PS/DVB) polymeric sorbent materials [47]. A scheme of the SPE sorbents and retention mechanism as a function of the analyte's properties is presented in Fig. 1.3. Obviously, some analytes can be extracted using different approaches, and selection of the most suitable extraction must take into account many factors, like experience with the specific SPE technique, simplicity of the procedure, possibilities of extending the method toward other analyte classes, and, of course, cost.

Regarding this last aspect, cost, classical silica-bonded reverse phase (RP) materials (C-18, C-8, etc.) are clearly advantageous. Nevertheless, its application towards 
(a)

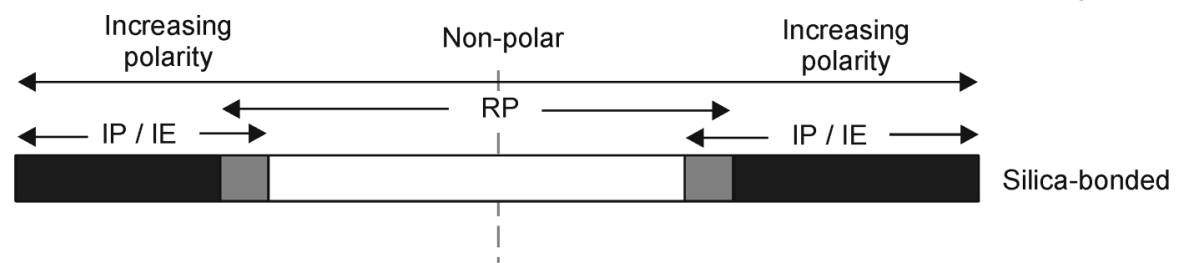

(b)

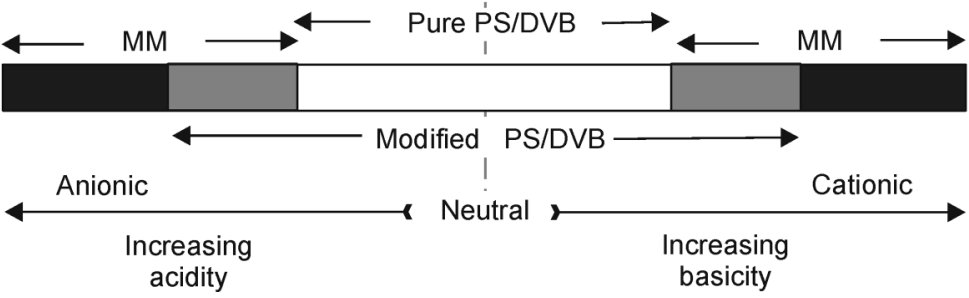

Polymeric

Fig. 1.3 Scheme of SPE sorbents and retention mechanisms for extracting polar pollutants from water as a function of analyte properties. IP: ion pair, IE: ion exchange, RP: reverse phase, PS/DVB: polystyrene/divinylbenzene, MM: mixed mode.

polar species is restricted to weakly acidic or basic compounds, which can be brought into the neutral species by adjusting the sample $\mathrm{pH}$. Thus, C-18 cartridges and disks have been successfully employed for the extraction of acidic drugs [10, 13, 48 ] and pesticides [3] by adjusting the sample $\mathrm{pH}$ to 2-3. However, recoveries of the most polar drugs and their metabolites (e.g., salicylic acid, hydroxy-ibuprofen...) are often incomplete [10, 13, 48]. Furthermore, $\mathrm{pH}$ adjustment of samples is limited by the stability of the silica. Therefore, this strategy cannot be applied to strongly acidic or basic analytes or to permanently charged species (e.g., amphoteric or quaternary ammonium compounds). Other problems encountered with silica-based RP materials are the residual silanol groups, which can interact with these analytes even when end-capped cartridges are used, and traces of metals in the silica if compounds with complexing properties (e.g., tetracyclines) are to be determined. In the case of tetracyclines, the problem is solved by adding EDTA to the sample [49].

The first approach to extracting strongly acidic or basic compounds by SPE was the use of ion exchange (IE) SPE [50]. Thus, as mentioned, one of the US-EPA methods for the determination of haloacetic acids is based on anion exchange SPE [43], where the sample $\mathrm{pH}$ is adjusted to 5 . The extraction of these compounds by an RP SPE employing silica-based materials would not be possible as the sample would need to be acidified to $\mathrm{pH} 0.5$ [44], where the silica bonds would be hydrolyzed. Another official method that relies on IE-SPE is the determination of complexing agents in water samples [51]. Here the International Standards Organization offers two possibilities: either evaporation of the water sample to dryness or IP-SPE before their derivatization and GC determination. Obviously, water evaporation requires a high temperature and is a time-consuming process, while IE-SPE can provide not just preconcentration but also a clean-up that evaporation cannot.

Other applications include quaternary ammonium and acidic herbicides [3, 4]. Yet, IE-SPE has some drawbacks, the major one being that recoveries are strongly affected by the ionic strength of the sample [4]. Therefore, strong matrix effects 
may occur in IE-SPE of environmental samples, where this parameter may change from sample to sample. For example, it was observed that the recoveries of the complexing agents NTA and EDTA decreased by 20 and $45 \%$, respectively, when $60 \mathrm{mg} \mathrm{L}^{-1}$ of sulfate was added to the sample [52].

The other way to extract very polar analytes on silica-bonded phases is to use ionpair (IP) reagents with RP materials (e.g., C-18), avoiding in this way the use of IE materials, facilitating the adaptation of conventional RP methods and allowing the combined extraction of a wide range of polarities. The retention of analytes can be tuned by selecting the chain length of the IP reagent, as a wide range of these are available (Table 1.1) for both basic/cationic and acidic/anionic compounds. Some of these ion-pairing agents are volatile enough to be compatible with LC-MS. Furthermore, the ion-pairing agent can suppress interactions with silanol groups of the sorbent [3]. Applications of IP-SPE in water analyses include the determination of acidic and quaternary ammonium herbicides [3, 4], acidic phosphoric acid mono- and diesters [53], and acidic pharmaceuticals and their microbial metabolites [54]. IP-SPE is the US-EPA official method for the determination of diquat and paraquat in drinking water [55] by using a C-8 disk and sodium 1-hexanesulfonate as IP reagent for retention; the analytes are then eluted by an $\mathrm{HCl}$ acidified solution, which breaks the IP. The technique of IP-SPE was reviewed by Carson in 2000 [56], who nevertheless recognized that this approach was seldom used for the SPE of polar compounds, in spite of the common use of IP formation for improvement of HPLC retention of polar compounds. A reason for this may be the fact that polymeric materials have had more success for SPE than for LC and a much wider chemistry is also available for SPE. In any case, IP-SPE can also be combined with polymeric materials, and it proved useful for the reduction of phenol breakthrough

Tab. 1.1 Common ion pair reagents. Partially adapted from [56].

For basic/cationic analytes $\quad$ For acidic/anionic analytes

Trifluoroacetic acid ${ }^{[\mathrm{a}]}$

Pentafluoropropionic acid ${ }^{[\mathrm{a}]}$

Heptafluorobutyric acid ${ }^{[\mathrm{a}]}$

Propanesulfonic acid salts

Butanesulfonic acid salts

1-Pentanesulfonic acid salts

1-Hexanesulfonic acid salts

1-Heptanesulfonic acid sats

1-Octanesulfonic acid salts

1-Nonanesulfonic acid salts

1-Decanesulfonic acid salts

1-Dodecanesulfonic acid salts

Dodecylsulfate, sodium salt

Dioctylsulfosuccinate, sodium salt

Bis-2-ethylhexylphosphate

\author{
Ammonia $^{[\mathrm{a}]}$ \\ Triethylamine ${ }^{[a]}$ \\ Dimethylbutylamine ${ }^{[a]}$ \\ Tributylamine ${ }^{[\mathrm{a}]}$ \\ Tetramethylammonium salts \\ Tetraethylammonium salts \\ Tetrapropylammonium salts \\ Tetrabutylammonium salts \\ Tetrapentylammonium salts \\ Tetrahexylammonium salts \\ Tetraheptylammonium salts \\ Tetraoctylammonium salts \\ Hexadecyltrimethylammoinum salt \\ Decamethylenbis(trimethylammonum bromide)
}

a LC-MS compatible 
on PS/DVB materials, thus allowing an enrichment factor increase by augmenting the sample volume [57].

A second attempt to improve the retention of polar analytes was the use of graphitized carbon black (GCB) and porous graphitic carbon (PGC) materials. These materials are able to retain compounds with a wide range of polarity, from very hydrophobic (e.g., PCBs) to very polar (e.g., some pesticides), and selectivity is gained by selection of the appropriate eluting solvent [58]. However, irreversible adsorption appears to be a frequent phenomenon, and selection of the appropriate eluting solvent is difficult as the retention mechanisms are still not very clear [58]. Carbon materials have been used for the extraction of several compound classes, alone $[3,4]$ or in combination with other sorbents [59]. Anyhow, the elution problems, together with the fact that sometimes low breakthrough volumes were reported [60], have hindered their popularization.

Major progress in the SPE of polar analytes came with the development of PS/DVB materials. These do not possess a silica core, and therefore all the associated problems ( $\mathrm{pH}$ limitation, residual silanol groups and metals and the need for SPE conditioning) were overcome. They also offer $\pi-\pi$ interactions, which increase the retention of some polar compounds [47]. Furthermore, this PS/DVB copolymer can be chemically modified by introducing more polar groups. Some researchers have investigated the introduction of vinylpyridine or vinylimidazole functional groups [61, 62], and many materials are now commercially available, like the IST Isolute ENV+ (hydroxy-modified PS/DVB), the Phenomenex Strata-X (modified PS/DVB patented composition) and the Waters Oasis HLB (polyvinlylpyrrolidone /DVB, Fig. 1.4). These polar groups increase the wettability of the sorbent and offer dipole-dipole and H-bonding interaction sites, improving the retention of polar analytes [63]. Of these materials, the Oasis HLB product appears to be the most extensively used sorbent for the extraction of polar compounds and especially acidic compounds, like herbicides [64], disinfection by-products [65], and acidic drugs $[66,67]$. It has also been employed for the SPE of neutral compounds like phosphoric acid triesters [68], weakly acidic analytes (e.g., triclosan [26, 27]),

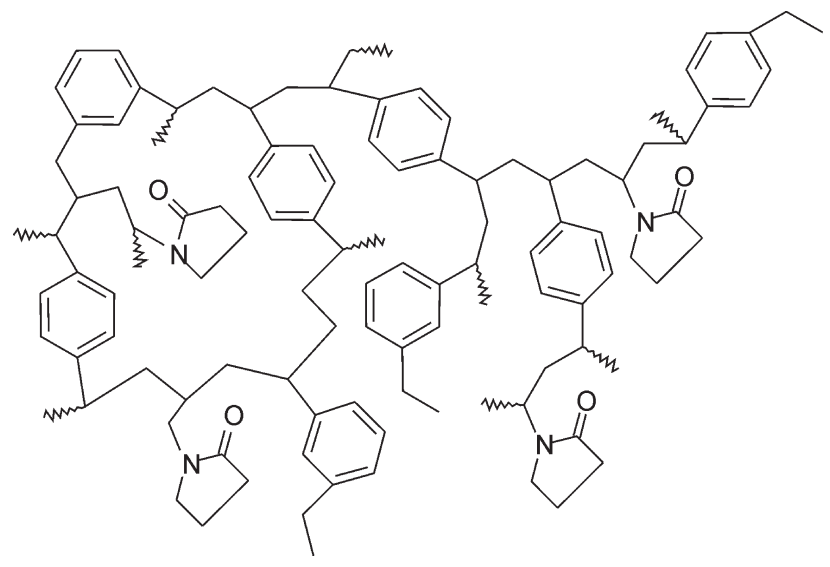

Fig. 1.4 Structure of the polymeric Oasis HLB sorbent. Redrawn from [50]; Copyright (C) 1998 John Wiley \& Sons. With permission of John Wiley \& Sons, Inc. 
and for the simultaneous extraction of acidic and basic compounds like benzothiazoles and benzotriazoles [69, 70]. In a recent study, several SPE sorbents were tested for the simultaneous extraction of acidic, neutral, and basic drugs, and Oasis HLB proved to provide the best recoveries even at $\mathrm{pH} 7.8$, thus avoiding the acidification step often necessary for the determination of acidic compounds with C-18 cartridges [71]. Furthermore, these sorbents can provide very high enrichment factors after $\mathrm{pH}$ adjustment, as no significant breakthrough was detected after the SPE of $2 \mathrm{~L}$ of pH 2 water for acidic herbicides [64] and pharmaceuticals [67]. However, this material is not suited for the extraction of permanently charged or very acidic or basic compounds. Here, IP-SPE or mixed-mode PS/DVB cartridges, where an ion exchange group has been introduced into the PS/DVB, or already modified-PS/DVB copolymer are required. These mixed-mode materials offer ion exchange groups to interact with the ionic parts of the analyte and the PS/DVB core for the more hydrophobic parts. This also allows the retention of a wider range of compounds. As an example, a mixed-mode anion exchange disk material has been proposed for the extraction of fluoroquinolone antibiotics in water samples [33]. On the other hand, the SPE of diquat and paraquat is achieved by using a mixed-mode cation exchange cartridge [72].

SPE can be coupled on-line with the LC(-MS) system, allowing the transfer of the whole extract into the chromatographic system, thus either reducing the sample volume sample that has to be extracted or increasing the method sensitivity. Such online SPE-LC-MS systems are technically more complex and require a careful adaptation of SPE elution and liquid chromatography. But given their high sensitivity and high reproducibility, with almost complete elimination of the risk of contamination, such systems are highly attractive when large numbers of samples have to be routinely analyzed for a fixed set of pollutants. Thus, most published applications are related to the determination of pesticides [7, 73, 74]. Enrichment and chromatographic separation of the analytes may also be achievable on one short column [75].

Finally, the future development of molecularly imprinted polymers (MIP) is expected to lead to an interesting alternative when a very specific SPE step is desired. These materials are synthesized in the presence of a molecule analogous to the target analyte, so that the synthesized MIP will have specific cavities and interacting sites suitable for interaction with a specific molecule or class of compounds [76]. Up to now, they have mostly been applied as a clean-up step, because the specific interactions take place under apolar organic solvent media. However, the direct application of water samples has already been demonstrated for the selective extraction of phenols [77, 78], where first the analytes are retained in an unspecific way on the MIP and then a non-polar solvent is applied to it, washing the interferences off while the analytes are retained. Despite some promising results, MIP still has to prove its broader applicability for analyte enrichment.

\subsubsection{Microextractions}

SPE has already made remarkable progress compared to LLE in terms of solvent consumption and automation. A step further was achieved by solid-phase microex- 
traction (SPME) and liquid-phase microextraction (LPME), where either no organic solvent is employed (SPME) or only a few microliters (LPME), and also the amount of sample is reduced to ca. 10-50 $\mathrm{mL}$ [45].

SPME was developed in 1989 [79] and became commercially available in 1993. In this technique, the analytes are first concentrated into a sorbent coated on a fusedsilica fiber that is exposed directly to the sample (direct sampling) or to its headspace (headspace sampling). After partitioning into this sorbent, the analytes can be desorbed either thermally by immersing the fiber into a GC injector or by an organic solvent if they are to be analyzed by LC or CE [80, 81]. Moreover, SPME can easily be automated for GC analysis. In the case of LC, automation can be achieved by so-called in-tube SPME, where the sample analytes are preconcentrated into a coated capillary that is connected on-line to the LC [82]. However, SPME hyphenation to LC is hampered by the slow desorption process, leading to band-broadening problems [45].

Nowadays, there are several sorbents available which cover a wide polarity range, and the main difficulty of the analysis of polar compounds relies on the need for derivatization of many of these compounds prior to their determination by GC. Thus, though the extraction of some polar compounds was possible using SPME, it yielded poor detection limits for many compounds due to poor chromatographic properties [83]. However, several options are available for combining SPME and derivatization, depending on the characteristics of the analytes and the derivatizating agent (Fig. 1.5) [45, 80, 84].

The most frequently employed method $[45,84]$ consists of adding the derivatizing agent directly to the sample. Thus, the desired derivatives are formed first and are then extracted into the SPME fiber by direct or headspace sampling for analysis. An example of this is the determination of basic psychiatric pharmaceuticals in wastewater by acetylation-SPME followed by GC-MS determination, where detection limits ranging between 15 and $75 \mathrm{ng} \mathrm{L}^{-1}$ for $10 \mathrm{~mL}$ sample volumes were obtained [85]. Another example is the determination of 18 aromatic primary amines,

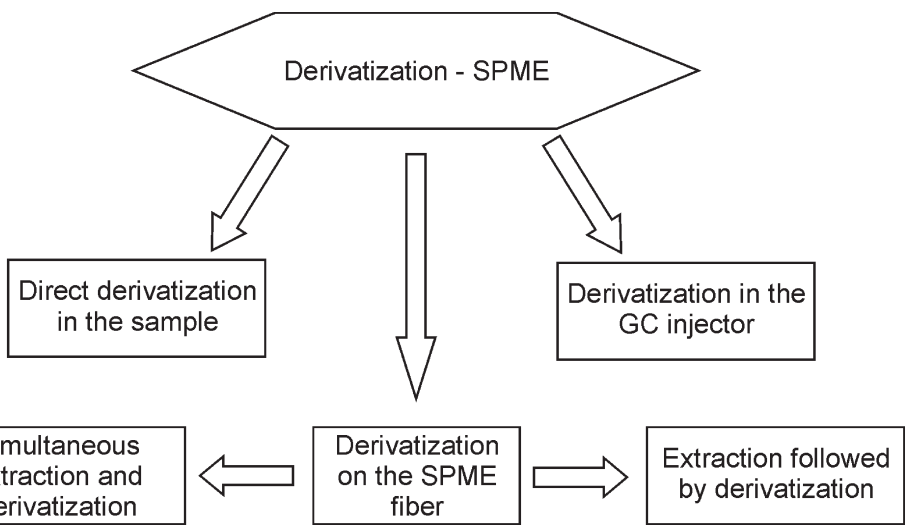

Fig. 1.5 Possible combinations of SPME and derivatization. Redrawn from [80], with permission from Elsevier. 
which were converted into their iodo-derivatives in a two-step process, further extracted by SPME, and then analyzed by GC-MS [86]. The substitution of the amine by an iodine group not only reduced the polarity of the amines, but also increased the mass of the analytes by $111 \mathrm{amu}$ (atomic mass units). This was advantageous for the mass spectrometric determination. However, the major limitation of the approach using direct derivatization in the sample is that it is not applicable to moisture-sensitive reactions.

The most popular method of avoiding this inconvenience is to first extract the analytes by SPME and then to derivatize them by exposing the fiber to the vapors of a derivatizing agent. In this way, SPME can be combined with silylation reactions, for example, to generate tert-butyldimethylsilyl-derivatives of triclosan and their related degradation products [87], for acidic drugs [88] or acidic herbicides [89]. This combination led to detection limits between 2 and $40 \mathrm{ng} \mathrm{L}^{-1}$ from sample volumes of about $20 \mathrm{~mL}$. Figure 1.6 shows the GC-MS chromatogram of a wastewater sample containing $45 \mathrm{ng} \mathrm{L^{-1 }}$ of mecoprop obtained by SPME extraction and on-fiber derivatization [89].

The other two combinations of derivatization and SPME shown in Fig. 1.5 (namely, "simultaneous extraction and on-fiber derivatization" and "derivatization

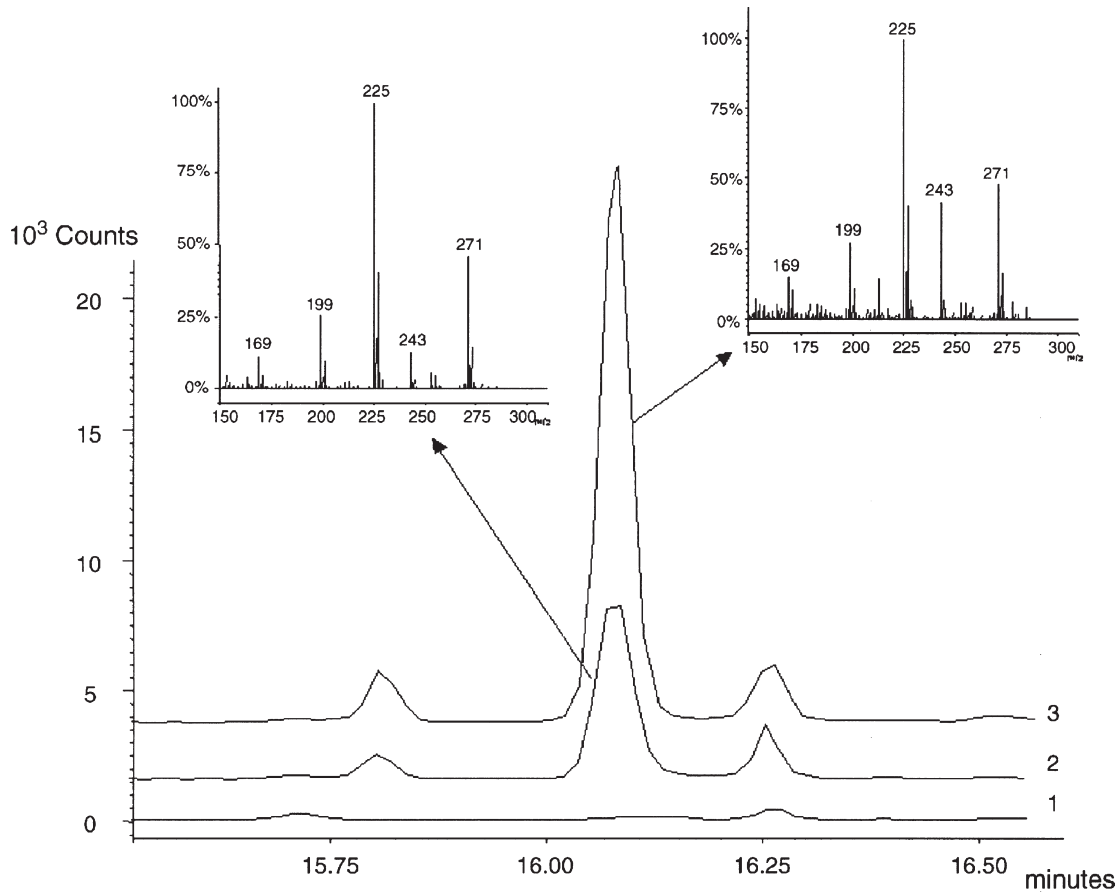

Fig. 1.6 SPME-GC-MS overlay of chromatographic signals (traces at $m / z 225+227$ ) and MS spectra of mecoprop in a blank sample (1), a non-spiked sewage water $(46 \mathrm{ng} / \mathrm{L})$ (2) and a sewage water spiked with the compound at $180 \mathrm{ng} \mathrm{L}^{-1}$ (3). Reprinted from [89], with permission from Elsevier. 
in the GC injector") are only suitable for the analysis of polar organic analytes in very few concrete cases [45].

LPME is more recent than SPME and is based on partition of the analytes between the sample and a small volume (a few $\mu \mathrm{L}$ ) of an acceptor solution [45]. This can be carried out by direct contact between the phases [90] or by using a porous membrane as an interface [91-93], which appears to be more robust. It is also possible to employ either flow [93] or rod configuration [91, 92] and to perform the extraction as a two-phase or three-phase system (Fig. 1.7). In the two-phase system (Fig. 1.7i), the organic solvent fills the hollow fiber and the pores of the membrane, acting both as interface and extraction medium. In the three-phase system (Fig. 1.7ii) the organic solvent is used to impregnate the membrane pores and to act as the interface, while the acceptor solution is aqueous, like the sample. Enrichment is achieved by adjusting the sample and acceptor $\mathrm{pH}$ in such a way that the analyte solubility is reduced in the sample and enhanced in the acceptor. For example, for the analysis of acidic analytes, the sample is acidified to obtain the neutral species, which are then extracted to the organic interface and finally back-extracted to the acceptor solution, adjusted to a basic $\mathrm{pH}$, so that analytes are trapped in their anionic form. With this three-phase LPME, a very selective extraction is achieved, which may eliminate the commonly encountered matrix effects for the LC-MS/MS analysis of environmental samples [94], provided that analytes have some acidic or basic function. Up to now, home-made devices had to be used for LPME, resulting in low reproducibility and difficult handling, but a commercial device is now available (Gerstel, Mühlheim an der Ruhr, Germany), which, however, uses larger amounts of solvent (ca. $0.5-1 \mathrm{~mL}$ ).

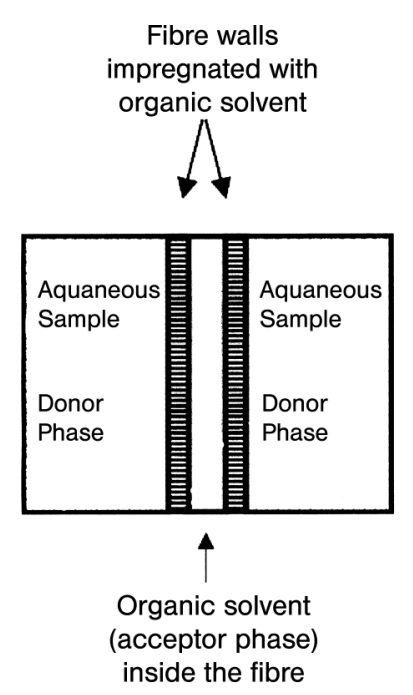

(i) Two-phase system
Fibre walls impregnated with organic solvent
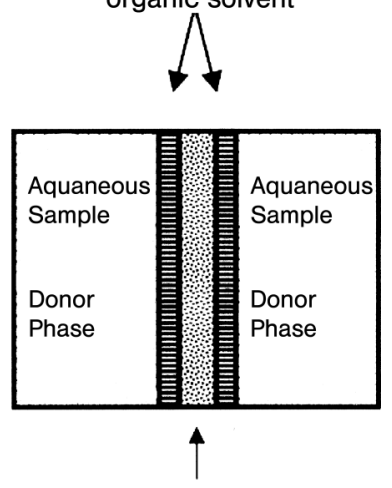

Aqueous solution (acceptor phase) inside the fibre

(ii) Three-phase system

Fig. 1.7 Cross section of the hollow fiber inside the aqueous sample during (i) two-phase and (ii) three-phase LPME. Reprinted from [92], with permission from Elsevier. 
1.4

\section{Gas Chromatographic Methods}

The GC determination of polar analytes covered in this book in most instances requires a prior derivatization step, with some exceptions, i.e. neutral compounds such as trialkyl phosphates, neutral drugs and disinfection byproducts. Yet even this is not possible for some analytes, and these are usually determined by LC (normally LC-MS/MS, see Section 1.5) or CE. Despite this and the high sensitivity obtainable by LC-MS/MS systems in the last decade, GC still offers some clear advantages over LC, primarily higher separation efficiency and lower costs without the problems associated with matrix effects of LC-MS/MS.

\subsection{1}

\section{Derivatization}

A really wide variety of derivatization reactions are available for many analyte classes. We only discuss the most important of these here, pointing out their advantages and disadvantages and giving some examples, although some have already been mentioned in Section 1.3. Table 1.2 presents an overview of the most common. More details can be found in several books and reviews [9, 95-100].

Tab. 1.2 Overview of most common derivatization reagents.

\begin{tabular}{|c|c|c|c|}
\hline $\begin{array}{l}\text { Reaction } \\
\text { type }\end{array}$ & Reagent & $\begin{array}{l}\text { Aqueous } \\
\text { media } \\
\text { compatible }^{[a]}\end{array}$ & Target functional group \\
\hline \multirow[t]{5}{*}{$\begin{array}{l}\text { Alkylation/ } \\
\text { Esterification }\end{array}$} & Diazomethane & NO & $\begin{array}{l}-\mathrm{COOH} \text {, acidic }-\mathrm{OH} \\
\text { and }-\mathrm{NH} \text { group }\end{array}$ \\
\hline & (Perfluoro)alkyl halides & NO & $\begin{array}{l}-\mathrm{COOH} \text {, acidic }-\mathrm{OH} \\
\text { and }-\mathrm{NH} \text { groups }\end{array}$ \\
\hline & $\begin{array}{l}\text { Quaternary ammonium/ } \\
\text { sulfonium salts }\end{array}$ & NO & $\begin{array}{l}-\mathrm{COOH} \text {, acidic }-\mathrm{OH} \\
\text { and }-\mathrm{NH} \text { groups }\end{array}$ \\
\hline & Alcohols & NO & $-\mathrm{COOH}$ \\
\hline & (Perfluoro)acyl chloroformate & YES & $-\mathrm{COOH}$ \\
\hline \multirow[t]{3}{*}{ Acylation } & (Perfluoro)acyl halides & NO & $-\mathrm{OH}$ and basic $-\mathrm{NH}$ groups \\
\hline & (Perfluoro)acyl anhydrides & YES & $-\mathrm{OH}$ and basic $-\mathrm{NH}$ groups \\
\hline & (Perfluoro)acyl chloroformate & YES & $-\mathrm{OH}$ and basic $-\mathrm{NH}$ groups \\
\hline \multirow[t]{2}{*}{ Sylilation } & BSTFA, MSTFA & NO & $\begin{array}{l}\text { - } \mathrm{COOH},-\mathrm{OH} \text {, acidic } \\
\text { and basic-NH groups }\end{array}$ \\
\hline & MTBSTFA & NO & $\begin{array}{l}-\mathrm{COOH} \text {, acidic }-\mathrm{OH} \\
\text { and } \mathrm{NH} \text { groups }\end{array}$ \\
\hline
\end{tabular}

\footnotetext{
a "NO" means that the derivatization must be carried out in an organic solvent
} 


\subsubsection{Alkylation and Esterification}

This reaction consists of the replacement of an acidic or slightly acidic hydrogen by an alkyl group, yielding the corresponding ether or ester. Among the alkylating reagents, diazomethane is probably the most common because of its high reactivity toward most acidic groups, including carboxylic acids and several hydroxyl groups. It has been widely employed, for example, in the determination of acidic pharmaceuticals and herbicides $[10,66,101,102]$ or phenolic analytes, like triclosan [26]. However, diazomethane is toxic, carcinogenic and potentially explosive if not handled with care and must be stored at $-20^{\circ} \mathrm{C}$. Hence, many other alkylation reagents have been investigated as potential replacements.

For example alkylation may be performed with alkyl halides in the presence of a base catalyst. The most frequent reagent of this type is pentafluorobenzyl bromide (PFBBr), which produces an electron-rich derivative that can be sensitively analyzed by GC-ECD or GC-NCI-MS. A good example of this is the determination of several phenolic endocrine disruptors (estrogens, surfactants, etc.) by SPE, PFBBr derivatization and GC-NCI-MS, resulting in detection limits at the $20-200 \mathrm{pg} \mathrm{L}^{-1}$ level, depending on the sample matrix and the volume employed for extraction (ranging from 1 to 5 L) [103]. Although this procedure cannot be carried out in aqueous media, it tolerates small amounts of water and can be directly coupled to the procedure for the extraction of dried solid samples, as in the PLE of acidic herbicides from soil [39], which has already been mentioned.

On-column or in-port alkylation can be accomplished by tetramethylammonium hydroxide (TMAH) or trimethylsulfonium hydroxide (TMSH) and other alkyl analogs, which undergo pyrolytic decomposition at the GC injection temperature, donating alkyl groups (methyl groups in these cases) and yielding trimethylamine or dimethylsulfide, respectively, as decomposition by-products [104]. While TMAH may damage the analytical column, TMSH is less reactive. They can also be used as ion-pairing reagents in order to enhance analyte extractability, for example, in combination with SPME for the determination of linear alkylbenzenesulfonates [105] and perfluorocarboxylic acids [106]. They have also been used for the derivatization of acidic pharmaceuticals and their metabolites after SPE [107-109]. Because of the limited stability of the products, analysis on the same day as the TMSH addition was recommended [108].

Carboxylic acids can also be esterified with an alcohol or by the use of alkylchloroformates. The latter compounds in fact act as alkylating agents with carboxylic acids and as acylating agents with amines and alcohols. They have the advantage that the reaction takes places even under aqueous conditions, so that derivatization can be performed on the sample before extraction [96, 110]. This derivatization scheme is also used in LC determinations to introduce a readily detectable fluorescent group or to increase the retention of analytes in the LC column (see Section 1.5.1.3). However, these reactions are not always easy to perform because they suffer from matrix effects affecting the derivatization yield [111]. 


\subsubsection{Acylation}

Acylation is normally the derivatization of choice for amines, and it is also very common for phenolic compounds, yielding either amides or esters. The most popular reagents are acyl halides, acyl anhydrides, and their corresponding perfluorinated analogs, when ECD or NCI-MS detection is desired. These reagents are compatible with moisture, so that the derivatization can be carried out prior to extraction in aqueous solution.

The example of the acetylation of basic drugs combined with SPME has been already discussed [85]. Acetylation has also been employed in the determination of phenolic compounds in water samples by SPE and SPME [112, 113].

\subsubsection{Silylation}

Silylation is the most widely used derivatization technique for GC analysis. This technique allows the derivatization of aromatic and also aliphatic alcohols, carboxylic acids, amines, and amides. Because of their reactivity, however, silylating agents are very sensitive to traces of water and other protic solvents.

There are two main classes of silylating agents: those producing trimethylsilyl (TMS) derivatives and those producing tert-butyldimethylsilyl (TBDMS) derivatives. TMS derivatives are more common and can be produced by a wide variety of reagents, like $\mathrm{N}$,O-bis-trimethylsilyl-trifluoroacetamide (BSTFA) and $\mathrm{N}$-methyl- $\mathrm{N}$ trimethylsilyl-trifluoroacetamide (MSTFA). Trimethylsilylation has been applied to many polar contaminant classes, including phenols [114], acidic herbicides [114] and acidic pharmaceuticals $[17,115]$. The derivatization reaction can also be performed directly on SPE disks [114], producing better yields than derivatization with alkyl halides or methanol/BF 3 .

TBDMS derivatives are prepared by reaction with $N$-tert-butyldimethylsilyl-trifluoroacetamide (MTBSTFA). Compared to TMS-reagents they are less sensitive to moisture and tolerate about $1 \%$ of water [64]. Moreover EI-MS spectra of TBDMSderivatives are normally characterized by a base peak corresponding to the loss of the tert-butyl (57 amu) group from the molecular ion. This helps in the identification of unknown compounds and in the confirmation of analytes by MS spectra (Fig. 1.6). Derivatization with MTBSTFA has been applied to acidic herbicides [64, 89], acidic pharmaceuticals [67, 88], phenolic compounds [87, 116], and amide type drugs [117]. This versatility is, indeed, one of the advantages of silylation reagents, as they can derivatize a wide range of compounds, including amide groups. In a comparison by Reddersen and Heberer [118] between MTBSTFA and PFBBr for the derivatization of several polar compounds and determination by GC-EI-MS, it was concluded that PFBBr provides slightly better detection limits, but MTBSTFA is able to derivatize a wider range of analytes, including carbamazepine, a compound that otherwise partially decomposes in the GC injector.

However, the drawback of TBDMS reagents as compared to TMS reagents is their lower reactivity and larger size. Thus, it was found that MTBSTFA could derivatize only the aromatic -OH groups of estrogenic compounds, while BSTFA and MSTFA react both with aromatic and aliphatic-OH groups, but only MSTFA 
provides a complete derivatization of $\alpha$-ethynylestradiol and mestranol, because of the smaller size of MSTFA [119].

\subsection{2}

\section{Separation and Detection}

\subsubsection{Separation}

Gas chromatographic separation of non-polar analytes or derivatized polar analytes is normally carried out on non-polar, DB-1 or DB-5 type, columns without problems.

A special application is the separation of enantiomers, which may show different biodegradability in the environment. In earlier work, separation was achieved using laboratory-made columns, e.g., the separation of isomers of ibuprofen (after methylation), which was carried out by a column containing the chiral selector 2,6O-dimethyl-3-O-n-pentyl- $\beta$-cyclodextrin (DMPEn) [102]. For some acidic pesticides (after derivatization) a column containing heptakis-[2, 3-dimethyl-6-tert-butyldimethylsilyl]- $\beta$-cyclodextrin (TBDM $\beta$-CD) was used [101, 120]. Meanwhile, chiral GC columns became more accessible, and, for example, the separation of the pesticide metalaxyl has been accomplished on a PS086-BSCD column (BSCD: tert-butyldimethylsilyl- $\alpha$-cyclodextrin; BGBAnalytik, Adliswil, Switzerland). As shown in Fig. 1.8 , an acceptable resolution was obtained, that allowed to follow the enantiomerspecific biodegradation of this compound in soils [121].

Two very recent trends in GC are (a) the development of fast separations by the use of narrow-bore capillary columns and (b) the development of comprehensive two-dimensional gas chromatography (GCxGC). (A) Fast GC has already been applied to several hydrophobic compounds, but the application to polar analytes is still quite uncommon [122]. A method that combined SPE of $10 \mathrm{~mL}$ of water and fast GC-MS using a PTV injector allowed the determination of several pesticides at the ng $\mathrm{L}^{-1}$ level from a low sample volume and with a GC analysis time of less than 8 min [123]. Analysis times can be further reduced by so-called ultra-fast GC, which uses narrower columns and a specific heating system, in order to obtain reproducible high temperature ramps. TOF detectors can be coupled if MS detection is needed. An example of the separation of some pesticides by ultra-fast GC is shown in Fig. 1.9, where 14 analytes were separated in less than 2 min [124]. (B) GCxGC provides unsurpassed 2-dimensional chromatographic resolution and allows the separation of very complex mixtures. It has as yet been primarily applied to non-polar compounds (geochemistry, oil chemistry, PCBs, etc.) $[125,126]$. Recently GCxGC has been used to separate up to 102 nonylphenol isomers in river water [127].

\subsubsection{Detection}

Electron capture detectors (ECD) and nitrogen phosphorus detectors (NPD) are still in use. However, as these detectors cannot provide additional confirmatory information, a mass spectrometer has become the detector of choice. GC-MS systems are nowadays relatively inexpensive, and especially ion trap detectors (ITD) allow the GC-MS/MS analysis of complex samples (e.g., sludge) with low limits of 

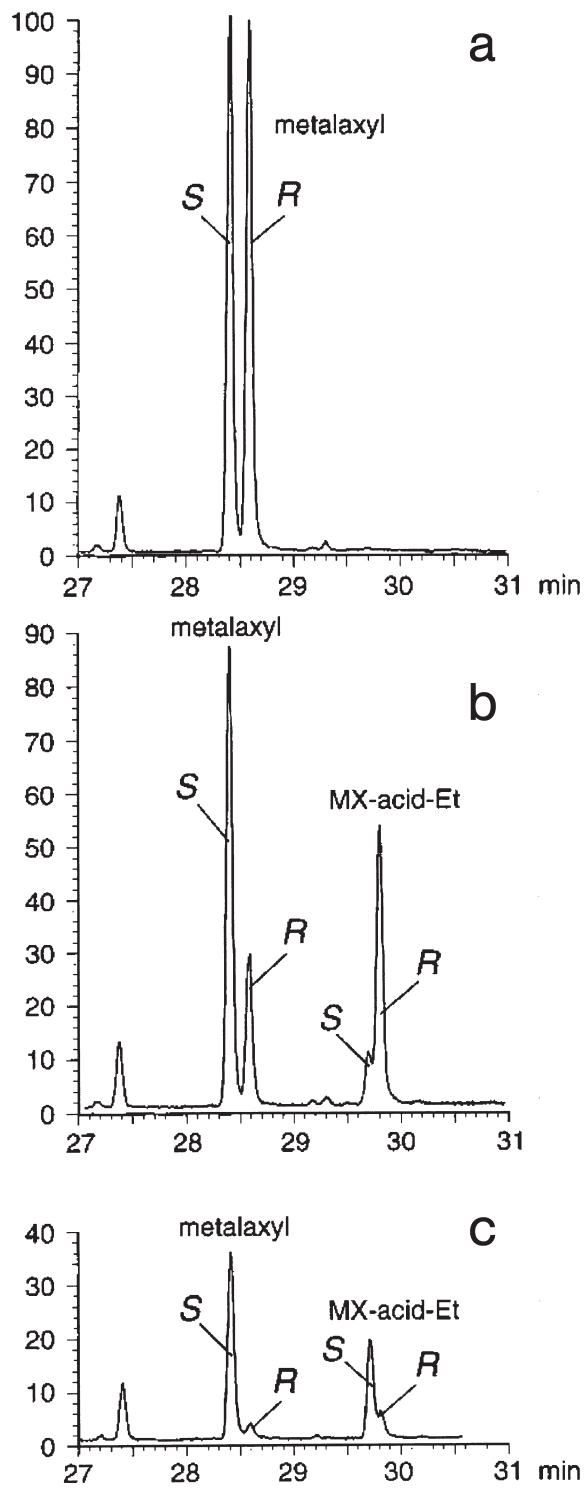

Fig. 1.8 GC EI SIM chromatograms $(m / z$ 220) showing elution of metalaxyl and MX-acid enantiomers (analyzed as the ethyl ester) from the incubation of racemic-metalaxyl in soil after (a) 0, (b) 28, and (c) 74 d, using the chiral PS086-BSCD column. Reprinted with permission from [121]. Copyright (2002) American Chemical Society.

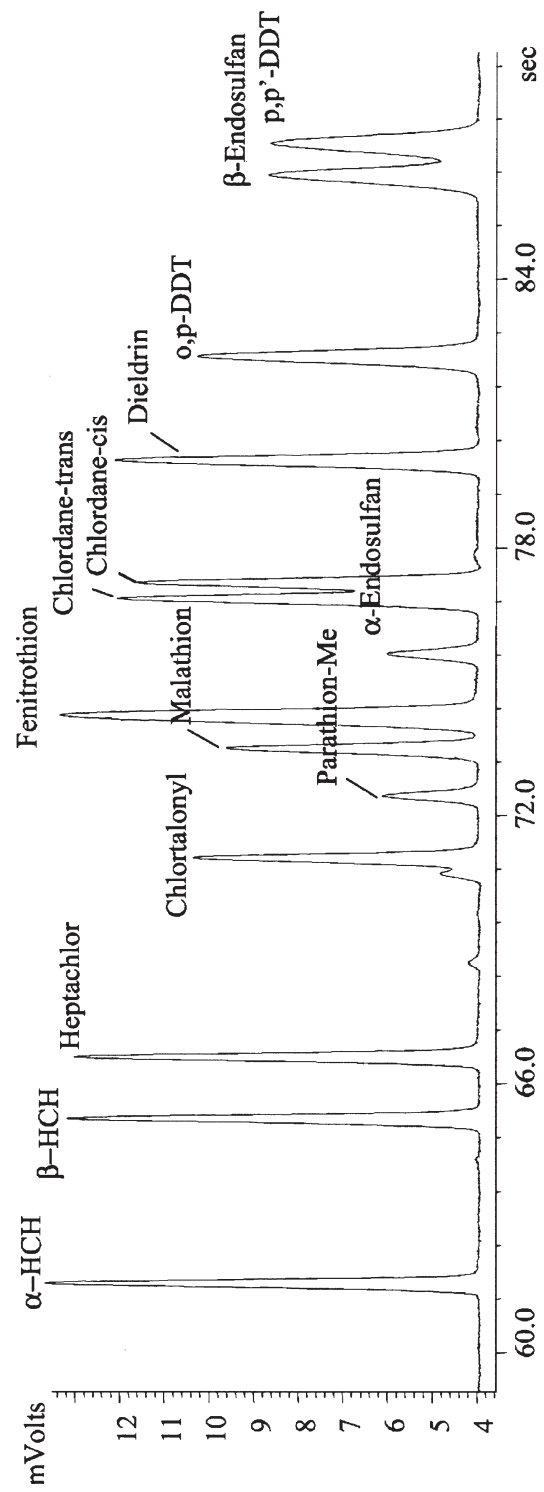

Fig. 1.9 Ultra-fast GC-FID pattern of a pesticide standard mixture. Note that the time axis is given in seconds. Reprinted from [124], with permission from Elsevier. 
detection. Thus GC-ITD-MS/MS has been applied to the determination of several pharmaceuticals [101, 128] and some nonylphenol polyethoxylates [129] in river and sea water, where higher sensitivity and selectivity are needed, providing additional structural information for the identification of their degradation products $[108,129]$. Another way of improving sensitivity and selectivity is by the use of negative chemical ionization (NCI) if the compounds have electrophilic groups. Sensitivity toward NCI can also be increased by forming perfluoro derivatives, e.g., for the determination of acidic pesticides in soil [39].

The use of programmed temperature vaporizers (PTV) or large volume injectors (LVI) is a final alternative for improving the detection limits. Actually, PTV / LVI are now commercially available and implemented in many GC systems and they can provide injection volumes higher than $100 \mu \mathrm{L}$, with the corresponding 100-fold increase in sensitivity [7] and it is expected that their use will be routine soon. However, it must be kept in mind that chemical noise increases in the same way and improved cleanup may be required.

\section{5}

\section{Liquid Chromatography-Mass Spectrometry}

In view of the chemical properties of polar pollutants, many of which are ionic and thermally labile, high-performance liquid chromatography (HPLC) would appear to be a more adequate chromatographic technique than gas chromatography. However, robust HPLC equipment was developed only as recently as the late 1980s. UV detectors, followed by diode-array detectors and fluorescence detectors, were most commonly employed for trace analysis by LC. Until the late 1990s, trace analysis by HPLC was hampered by the limited sensitivity and, more severely, by the limited selectivity of these detectors. Thus, much effort was spent to extend the polarity range of GC-based methods toward polar and ionic analytes, often by derivatization (see Section 1.4).

Since then, LC-based methods have rapidly gained ground in the determination of polar pollutants from aqueous samples as atmospheric pressure ionization (API) mass spectrometers came onto the market and were combined with liquid chromatography, resulting in robust and versatile LC-MS instrumentation.

LC, as well as being tailor-made for the separation of polar water-soluble analytes, offers two increasingly important advantages over GC-based methods. (A) An on-line coupling with solid-phase extraction is comparatively easy to achieve and has been widely used for many classes of compounds, especially pesticides [73, 74, 130, 131]. (B) LC allows aqueous samples to be directly injected into the chromatographic system. With the steadily increasing sensitivity of API-MS instruments, this is becoming more and more attractive.

The next section focuses solely on LC-MS-based methods and does not consider the UV, diode array, and fluorescence detectors used previously. More detailed reviews on the use of LC-MS for the trace analysis of polar pollutants have appeared in recent years [132-135]. 
1.5.1

\section{Liquid Chromatography}

There is a large body of literature on the LC separation of the various classes of polar contaminant. It must be noted, however, that the coupling of LC to MS poses some restrictions on the LC method, as completely volatile eluents are mandatory. Moreover, the composition of the LC eluent, its inorganic and organic modifier and its $\mathrm{pH}$, may interfere strongly with the ionization of the analytes of interest and may thus have a significant effect on the sensitivity of detection. For these reasons, traditional LC methods may not be appropriate for LC-MS analyses. Unfortunately, the influence of the eluent composition on the sensitivity of MS detection turned out to be difficult to predict, and any theory on this subject cannot be a substitute for trials (and errors).

Generally, the criteria used to select the appropriate chromatographic mode are the same as those used for selecting the mode of solid-phase extraction (see Fig. 1.3a in Section 1.3.3.1). At the extremes, ion chromatography (IC, with some limitations) or ion-pair reversed-phase liquid chromatography (IP-RPLC) may be used for strongly basic or acidic analytes, whereas reversed-phase LC (RPLC) is used for most other analytes, with $\mathrm{pH}$ adjustment, when weakly acidic or basic compounds are analyzed. Normal-phase chromatography has never played a significant role in trace analysis of polar pollutants, but hydrophilic interaction chromatography (HILIC) may fill this gap in the future.

\subsubsection{Ionic Analytes}

\section{Strongly Acidic Compounds}

Many environmentally relevant strongly acidic compound classes are sulfonated aromatic compounds. This functional moiety occurs in technically used naphthalene mono- and di-sulfonates (NSA and NDSA), in sulfonated naphthalene formaldehyde condensates (SNFC), in anionic tensides like linear alkylbenzene sulfonates (LAS; see Chapter 9), and in many azo dyes and their degradation products. Owing to the acidity of the sulfonate group, protonation to the non-ionic form by lowering the eluent's $\mathrm{pH}$ value is not possible. Extraction and chromatography of such polar aromatic sulfonates are usually based on ion-pair formation, with volatile cationic counter ions (Fig. 1.10). With ammonium acetate [136] or triethylamine [137], monosulfonates are sufficiently retained, while a strong retention even of trisulfonated naphthalenes has been obtained with tributylamine (TrBA) [138]. IP-RPLC is also suited for the LC-MS analysis of degradation products of azo dyes $[139,140]$ or sulfonated phthalocyanine dyes [141].

Although the sulfonate group of LAS is quite acidic, the long hydrophobic alkyl chain provides sufficient retention in RPLC, so that IP-RPLC need not be used. In RPLC, LAS mixtures are separated according to the alkyl chain length [142, 143]. However, retention of the more polar carboxylated degradation products of LAS, the sulfophenyl carboxylates (SPC), requires cationic counterions such as triethyla- 


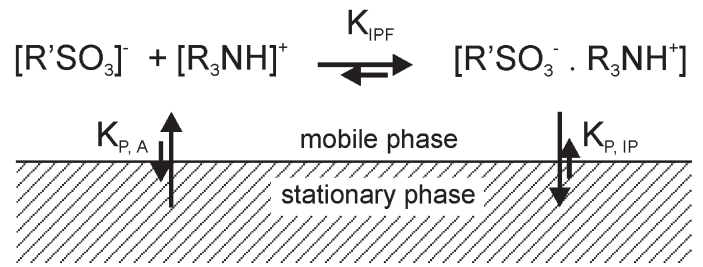

Fig. 1.10 Principle of ion-pair chromatography (IP-RPLC). The partition coefficient for the free acid anion $\left(\mathrm{RSO}_{3}{ }^{-}\right.$) from solution (mobile phase) into the stationary phase $\left(K_{P, A}\right)$ is much smaller than the partition coefficient for the ion pair $\left(K_{P}, I P\right)$. Eluent conditions should ensure that the equilibrium of ion pair formation $\left(\mathrm{K}_{\mathrm{IPF}}\right)$ is on the right hand side.

mine (TrEA) [143-145]. IP-RPLC with TrEA is also applicable for the analysis of branched alkylbenzenesulfonates [146] and of SPC together with their LAS precursors $[144,145]$. In this case, SPCs elute before the LAS.

Only recently it was shown that IP-RPLC with TrBA is also suitable for the determination of 13 strongly acidic phosphoric acid mono- and diesters, some of which are used as plasticizers and occur in municipal wastewater [53].

Haloacetic acids (HAA) are also very acidic compounds, with $\mathrm{p} K_{\mathrm{a}}$ values of 0.7 to 2.9. Here, dibutylamine [147] or TEA [148] have been used as ion-pairing agents. Aminopolycarboxylic acids such as EDTA used as complexing agents are important polar pollutants found in many compartments of the water cycle (see Chapter 7). LC-MS methods have been developed using ion-exchange chromatography [149-151]; however, because of the chemical differences between the eluent and the aqueous sample, the speciation of EDTA may be substantially altered and weak complexes may be destroyed [151]. The use of a suppressor (cation exchanger) post-column strongly improves the detection limits [151] and avoids formation of sodium adducts in the interface [149].

Generally, the strength of the chromatographic retention of acidic compounds in IP-RPLC can be adjusted by selecting trialkylamine with a certain number of aliphatic carbon atoms. As the amine must be volatile, a total number of 9 to 10 carbons appears to be the upper limit. The protonated trialkylamines do not tend to form adducts in modern API interfaces but act as H-donors. Therefore these amines diminish the risk of sodium adduct formation with strongly acidic analytes, and they suppress multiple charging of compounds with more than one acidic group. However, these H-donors may also influence the ionization process in ESI [152], decreasing the sensitivity of detection [138]. Thus, the concentration of alkylamines should be kept as low as is acceptable for the chromatographic retention.

\section{Less Acidic Compounds}

Many polar pollutants are weakly acidic compounds, namely aromatic and aliphatic carboxylic acids. Among these are many pesticides (see Chapter 6) and pharmaceuticals (see Chapters $2-5$ ). Moreover, carboxylic acids are intermediates in the microbial or chemical oxidation of many classes of environmental contaminants. For example, SPCs are formed from LAS (see Chapter 9), alkylphenol ethoxycar- 
boxylates (NPEC or OPEC) and dicarboxylates (CAPEC) from non-ionic alkylphenol ethoxylates (APEO: NPEO or OPEO). Oxanilic acid derivatives can be generated microbiologically from chloracetanilide herbicides $[153,154]$. Carboxylic acids are also generated as breakdown products of aromatic hydrocarbons and many other aromatic compounds. There are three strategies to attain good peak shape and to increase retention of weakly acidic compounds in RPLC.

1. Mostly, the $\mathrm{pH}$ value of the eluent is decreased until the analytes occur in their non-dissociated state. Formic acid or acetic acid may be used, depending on the $\mathrm{pH}$ required. This approach was used for the analysis of X-ray contrast agents [59], succinates [155], and naphthalene carboxylates [156].

2. The addition of acid and the transformation of an analyte anion into its non-dissociated state could decrease the signal intensity for the molecular anion using ESI in negative mode. Alternatively, ammonium acetate can be added. The ion pair formed from ammonium and the analyte anion exhibits good peak shape and dissociates easily in the interface to release the molecular anion. Acidic pharmaceuticals like non-steroidal antiinflammatory drugs have long been analyzed by GC-MS after derivatization. LC-MS, using ESI in the negative ion mode with ammonium acetate as inorganic modifier, is also well suited for this purpose [157] and has also been used for acidic X-ray contrast media [158] as well as for oxanilic acid derivatives of herbicides [153, 154].

3. If the carboxylic acid analytes are small and polar, the non-ionic molecule or ionpairs formed with ammonium experience only weak retention in RPLC. Then, organic amines in their protonated form can be added to utilize IP-RPLC. This strategy has been used for the LC-MS analysis of bile acid conjugates using dibutylamine [159], for X-ray agents with a (non-volatile) tetraalkylammonium cation [160], and for acidic pharmaceuticals (antiphlogistics and lipid regulators, Fig. 1.11) and their more polar metabolites with volatile TrBA [54, 161]. Highly polar low-molecular-weight carboxylates may also be separated by CE-MS [162] or IC-MS [163].

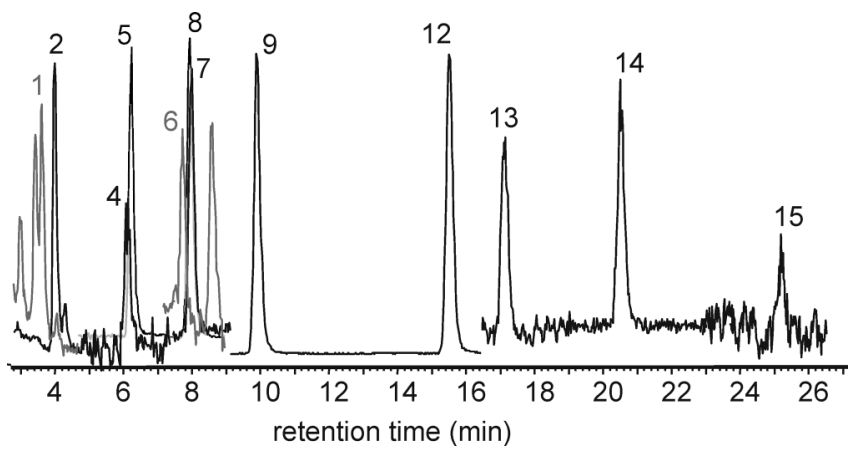

Fig. 1.11 LC-ESI-MS chromatogram of acidic pharmaceuticals in a municipal wastewater. IP-RPLC was used and analytes detected by MRM in negative ion mode. 1: salicylic acid; 2: 2,4-dichlorobenzoic acid (internal standard); 4: piroxicam; 5: clofibric acid; 6: keto- profen; 7: naproxen; 8: bezafibrate; 9: fenoprop (internal standard); 12: diclofenac; 13: indomethacin; 14: meclofenamic acid (internal standard) 15: triclosan. Redrawn from a chromatogram shown in [161]. 


\section{Strongly Basic Compounds}

It is fortunate that strongly basic compounds seldom occur in the aquatic environment, because the respective LC-MS approach of using anionic counterions to retain such compounds has not resulted in methods of satisfactory sensitivity. This is due to the fact that the polyfluorinated aliphatic carboxylic acids used as counteranions, namely hexafluorobutyric acid $[164,165]$ or pentafluoropropionic acid [166], strongly suppress the analyte signal in positive ion mode [167]. It was hypothesized that the analyte cations and the fluorinated carboxylate anions form an ion-pair that is too strong to dissociate in the interface. Maybe HILIC will be more successful than IP-RPLC for this application.

\section{Weakly Basic Compounds}

Quaternary ammonium compounds like dialkyl dimethyl ammonium salts or benzyl alkyl dimethyl ammonium salts are used as cationic surfactants and as anti-bacterial agents. Despite the ionic character of these compounds, their retention in RPLC is no problem because of the benzyl and long-chain alkyl substituents. However, a good peak shape is more difficult to obtain. Acidification reduces the tailing to some extent, and thus most eluents are acidified with formic acid [168, 169]. However, increasing acid concentrations may severely reduce the sensitivity of detection for basic nitrogenous compounds by ESI in the positive ion mode [170]. This may be because of the anionic counterions added with increasing acid concentration. These anions may unintentionally form ion pairs with the nitrogenous cations. The ion pairs are nonionic in solution and are thus less effectively transferred into the mass spectrometer [170]. If quaternary ammonium compounds become more polar, and retention in RPLC is difficult to obtain, IP-RPLC can be used (see above).

Aromatic nitrogenous compounds like benzothiazoles $[69,171]$ and benzotriazoles [70] can be analyzed with ammonium acetate using ESI in positive mode. The same approach is used for basic pharmaceuticals such as $\beta$-2-sympathomimetics and beta-blockers $[10,48,158]$. A method for the determination of carbamazepine and six metabolites with ammonium acetate at pH 4 was developed [172].

Sulfonylurea, imidazolinone, and sulfonamide herbicides are quite effective and need to be determined with high sensitivity. As for many other basic analytes RPLC with a formic acid ammonium formate buffer ESI in positive ion mode has been used to determine 16 of these analytes in surface and groundwater [173].

\subsubsection{Non-Ionic Analytes}

Alkylphenol ethoxylates (APEO) are the most important class of non-ionic surfactants (see Chapter 9), and many methods for their analysis by LC-MS have been developed. Since the hydrophobic part of APEO is identical for all components, all ethoxy homologs tend to coelute in RPLC separation [174-176], and the distinction between them is only made by means of the MS detection. This coelution has often been claimed to be advantageous, since it would increase the sensitivity of detection. As a matter of fact, quantitation is severely compromised by the coelution, 
because the response factors of homologs vary substantially, with a notoriously poor sensitivity for the monoethoxylate (e.g., NP1EO). Moreover, fragmentation of one ethoxy-homolog results in the increase of the response for the next lower homolog. This is not recognized and impairs quantification if these homologs are not chromatographically separated. So-called mixed-mode chromatography relying on size-exclusion and reversed-phase mechanisms provides full resolution of the homologs (Fig. 1.12) [177]. The chromatographic separation of the homologs avoids the interference of the MS signals of different homologs due to fragmentation and allows optimization the MS parameters for each of the homologs.

The chromatographic separation of alcohol ethoxylates (AEOs) by RPLC strongly depends upon the organic modifier used: with a water/methanol gradient, AEOs are separated according to the length of the hydrophobic alkyl chain [178-180], whereas a water/acetonitrile gradient also provides some separation according to the number of ethoxy groups $[155,180]$. Alkylpolyglycosides (surfactants based on sugars and fatty alcohols) have a hydrophobic alkyl chain of 8 to 16 carbons that mediates retardation on RPLC $[181,182]$.

Neutral pharmaceuticals are not a chemically homogenous class of compounds. They are rather a mixture of environmentally relevant compounds (e.g., carbamazepine, diazepam, cyclophosphamide) that can be analyzed together at neutral $\mathrm{pH}$ [183]. Similar conditions can be used for macrolide antibiotics (clarithromycin, erythromycin), which are, however, more hydrophobic and require increased organic modifier concentrations [48, 184].

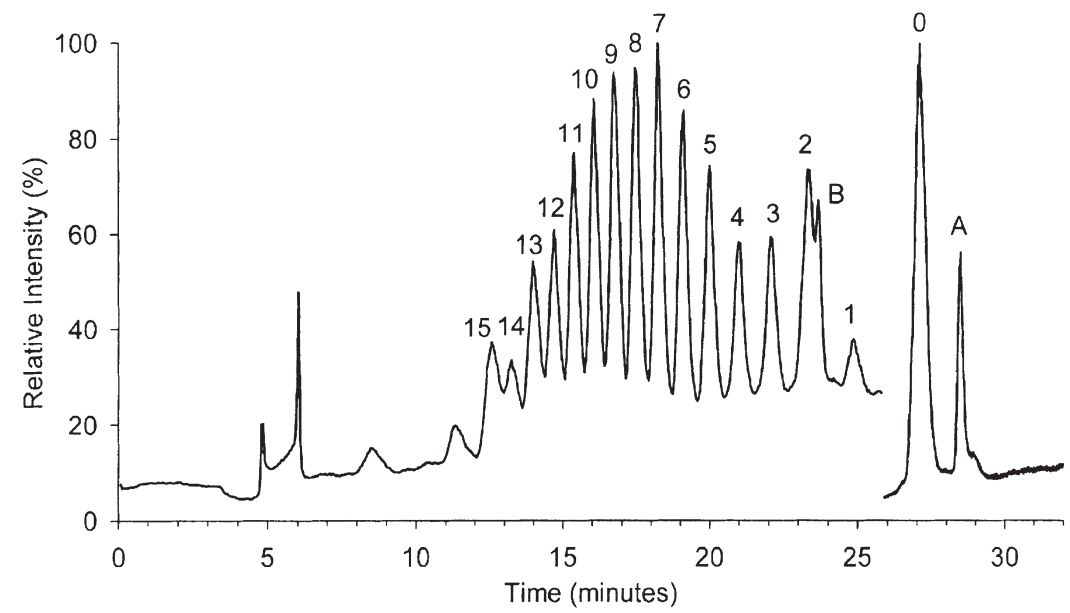

Fig. 1.12 LC-ESI-MS chromatogram of a technical mixture of NPEO. A mixedmode separation based on SEC and RPLC was used for chromatography. Numbers denote the number of ethoxylate groups in the respective NPEO homolog, 0 denotes the NP. A and B are internal standards. Reprinted from [177], with permission from Elsevier. 


\subsubsection{Amphoteric Compounds}

Many biologically active polar compounds contain nitrogenous and oxygenous functional groups and exhibit amphoteric properties. Their chromatographic separation with satisfactory peak shape can be difficult, as it may be impossible to find $\mathrm{pH}$ conditions at which these compounds appear as non-charged molecules. Instead they may occur as cations, zwitterions, or anions. In terms of chromatography the zwitterionic form is probably the worst.

In most cases, acidic eluents were chosen, and ESI in positive ion mode was employed for detection. This scheme was used for fluoroquinolones (norfloxacin, ciprofloxazin, and others) $[33,184]$ and for amphoteric cocamidopropylbetaine surfactants [155]. For the latter, IP-RPLC is also reported [185]. In order to increase the signal response of amphoteric beta-lactam antibiotics (penicillins), methanolysis to form a secondary amine was used. These exhibited a much higher signal response in the positive ion mode than that of their parent penicillines [186].

The analysis of amphoteric tetracycline antibiotics is hampered by their tendency to form stable metal complexes and to interact strongly with free silanol groups of glassware and silica materials. This can be prevented by adding EDTA before extraction (see Sections 3.2 and 3.3.1). Tetracyclines have $\mathrm{p} K_{\mathrm{a}}$ values between 3.3 and 9.5 [187] and should be chromatographically separated with the acidic moiety in the non-dissociated form using an acidic eluent [184, 188]. Oxalic acid may be added to the eluent in order to suppress interaction with the silica core of the stationary phase [184]. Tetracycline epimers can occur that are difficult to separate by LC [189]. Detection of tetracyclines may also be performed by APCI [189], but ESI is more frequently used.

Several methods exist that combine the analysis of tetracyclines with that of sulfonamides $[187,189]$. This seems consistent, as sulfonamide chromatography requires an acidic $\mathrm{pH}$ [190], as for tetracyclines, and ESI in positive mode is applied for both classes of analytes.

The herbicide glyphosate and its biodegradation product aminomethylphosphonic acid (AMPA) are among the most challenging analytes in terms of polarity. While glyphosate contains only three carbons per molecule, there is only one in AMPA. In the extraction of these compounds, chromatographic retention and detection have been equally difficult to achieve. Derivatization with FMOC is one strategy that circumvents these problems by introducing a fluorescent group that also increases the molecular size and hydrophobicity, and allows the use of classical RPLC coupled to MS using ESI in negative mode $[111,191]$. The FMOC derivative also shows a good peak shape, which is more difficult to obtain with the original analytes. On the other hand, this derivatization reaction is not exempt of problems (see Section 1.4.1.1). Alternatively, IC-MS of anions is applicable with a cation suppressor coupled between the column and the ESI interface [149]. As for other very polar and ionic compounds, CE-MS is applicable but its sensitivity is limited because of the very small injection volumes of a few nanoliters [192]. 


\subsubsection{Multiresidue Methods}

Because of the substantial improvements in mass spectrometers, multi-residue methods across a range of chemically different compound classes are gaining in importance. The increasing sensitivity of mass spectrometers allows sensitivity to be sacrificed by working at non-optimal conditions, thus finding a compromise between the optimal conditions of each of the different compound classes that are combined. This has not been possible before, when optimal conditions in terms of eluent composition and instrumental settings were required for each analyte to attain sufficient sensitivity. A second instrumental improvement with respect to multiresidue methods is the possibility of rapid switching between positive and negative ion mode within one analytical run.

Presently, multiresidue methods are best developed in those areas of application where large numbers of chemically diverse analytes are found and a strong need for regular monitoring exists, such as in residue analysis of pesticides. Here, a method for the determination of 39 acidic and basic pesticides with ESI in positive and negative mode was developed comparatively early [193]. Similar approaches, now using triple quadrupole mass spectrometry in multiple reaction monitoring (MRM) instead of SIM (see Section 1.5.2.2), have been published [73]. In quite recent work, more than 100 pesticides of 20 chemical classes were analyzed within 20 min run time using ESI in positive (> 90 compounds) and negative ion mode (10 compounds) [194]. In terms of chromatography, mixtures of $\mathrm{MeOH}$ and AcCN or even ternary gradients are frequently used for these purposes.

Also for the trace analysis of pharmaceuticals the number of multi-methods is increasing. A method for the determination of 27 compounds, endocrine disruptors, pharmaceuticals, and personal care products using ESI in positive and negative mode has been published [195], as well as a procedure to determine 13 antibiotics belonging to 5 classes, including sulfonamides, tetracyclines, fluoroquinolones, and macrolide antibiotics using ESI in positive-ion mode [196].

\subsubsection{Chiral Separation}

Many polar pollutants found in the aqueous environment contain one or more chiral centers, especially when biological effects are intended, as in the case of pesticides and pharmaceuticals. Biotransformation may differ for the enantiomers, and a change in the enantiomeric ratio along a water path can provide an indication of the importance of biotransformation processes. Therefore, chiral separation is a valuable analytical process to study the fate of such biologically active compounds in the environment, as shown for ibuprofen [102] and pesticides [121] (see Section 1.4.2.1).

Chiral centers occur in several carboxylates, such as profen non-steroidal-antiinflammatory drugs and the chemically closely related chlorophenoxypropionic acids, tetracyclines, beta-blockers, and many other classes of compounds. Enantiomeric separation is usually based on chromatography using a stationary phase that contains one enantiomer of a so-called chiral selector. The chiral center of the polar analyte is often close to one polar or ionic moiety. Most LC enantiomeric separ- 
a)

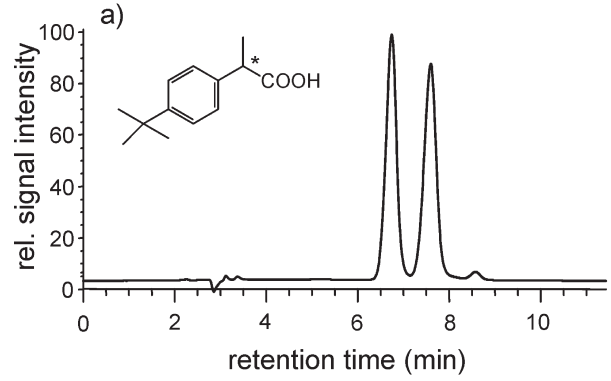

b)

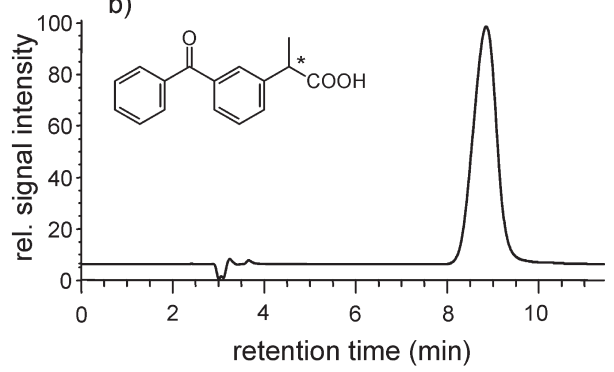

Fig. 1.13 LC-ESI-MS chromatogram of a separation of profen drug enantiomers on a chiral stationary phase (Chiralpak AD-RH using IP-RPLC). a) Ibuprofen; b) Ketoprofen. Quintana and Reemtsma, unpublished.

ations use normal-phase chromatography with only a few percent of water, because then the chiral selector can closely interact with the chiral center via the polar functional group. Several procedures of NPLC for the enantio-separation of profen drugs and beta-blockers are available [197, 198]. But, as mentioned before, NPLC is difficult to use for environmental samples: the sample solubility in pure organic solvent is often low, the samples often contain many polar matrix constituents that affect the lifetime of NPLC columns, and water may have to be added post-column to improve ionization in the MS interface. A few applications of RPLC for enantio-separation have been published, for example, for the separation of acidic profen drugs [199]. When RPLC is employed, however, hydrophobic interaction with the stationary phase governs the separation. If the hydrophobic part of a molecule becomes too large, then the influence of the polar chiral center may be too weak to obtain enantio-resolution. One such example is shown in Fig. 1.13: for ibuprofen an enantio-separation was achieved, whereas the enantiomers of the more hydrophobic ketoprofen could not be separated in IP-RPLC. To date, chiral separation of polar analytes using LC-MS is not a routine task in environmental analysis, and no applications to environmental samples are known.

Instead of liquid chromatography, capillary electrophoresis with cyclodextrins has frequently been used for enantiomeric separations, for example, for the phenoxyacid herbicides fenoprop, mecoprop, and dichlorprop [200].

\subsection{2}

\section{Mass Spectrometry}

\subsubsection{Ionization}

Two ionization interfaces, electrospray ionization (ESI) and atmospheric pressure chemical ionization (APCI), are available with most LC-MS instruments. There is also an ongoing effort to develop and to commercialize new interfaces, with the intention to extend the range of LC-MS toward less polar compounds that are more difficult to ionize. But, for the analysis of polar pollutants, this is quite irrelevant, and it appears that ESI and APCI will be the interfaces that accompany us for a long while. 
The overwhelming majority of literature on the determination of polar pollutants from water utilizes electrospray ionization (ESI), while APCI is used only rarely. This does not fully follow the general rule that compounds already appearing as ions in aqueous solution are best analyzed by ESI, whereas atmospheric pressure chemical ionization may be preferred for moderately polar compounds that are non-ionic in solution. This rule was generally confirmed in a detailed study by Thurman and coworkers comparing APCI and ESI for 75 pesticides (Fig. 1.14) [201]. They also showed that the composition of the eluent influences the signal response in ESI more strongly than in APCI and that adduct formation may be stronger in ESI. These latter two findings are in agreement with observations from real samples that the analyte response in APCI has been found to be less affected by coeluting matrix components than in ESI [202-205]. These are some arguments in favor of APCI.

However, when using ESI, matrix effects can be reduced by decreasing the flow directed into the ESI interface, which decreases the droplet size as well as the number of molecules to be ionized at a given time. Best effects appear to be obtained by using a nano-splitting device [206], but even a flow reduction to $50 \mu \mathrm{L} \mathrm{min}^{-1}$, which is possible with standard equipment, can diminish matrix effects [207].

As for the selection of ESI or APCI, the decision whether positive or negative mode works better is obvious at the chemically extreme ends. Bases are best detected as cations $\left([\mathrm{M}+\mathrm{H}]^{+}\right)$in positive mode and acids as anions $\left([\mathrm{M}-\mathrm{H}]^{-}\right)$in negative mode. Between these two extremes, however, there remains much scope for method optimization based on trial and error. Amphoteric compounds as well as many environmental contaminants that contain nitrogen and oxygen can be ionized in either mode, as has been found for sulfonamides and tetracyclines [189].

The final decision on whether ESI or APCI in positive or negative mode provides the best results depends not only upon the chemical properties of the analytes but also on the chromatographic system that is used, the eluent composition, and the "background" matrix of the samples that are to be analyzed. It is also important to note that the fragmentation pathway along which an ion breaks down is severely influenced by the charge it bears, as different functional elements of a molecule stabilize a positive or a negative charge. For an amino-hydroxy-naphthalene sulfonate, the transition $\mathrm{m} / \mathrm{z} 238 \rightarrow 80$ was most intensive in the negative ion mode, as compared to

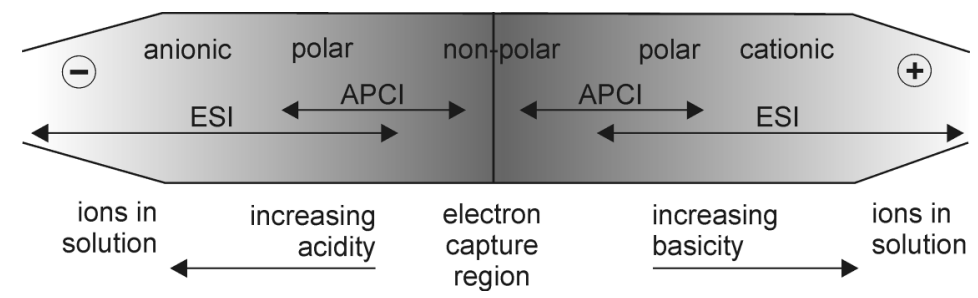

Fig. 1.14 Scheme of ionization modes (APCl: atmospheric pressure chemical ionization; ESI: electrospray ionization) selected as a function of analytes properties. Reprinted with permission from [201]. Copyright (2001) American Chemical Society. 

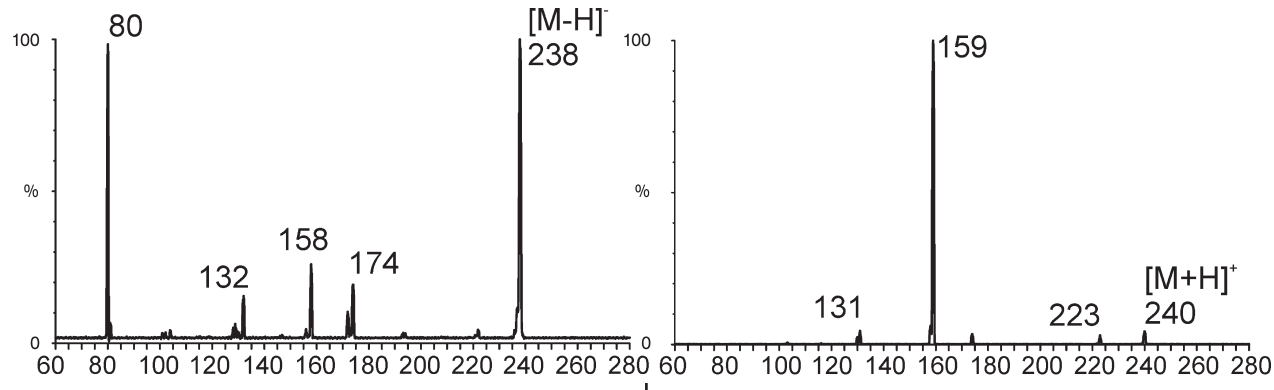

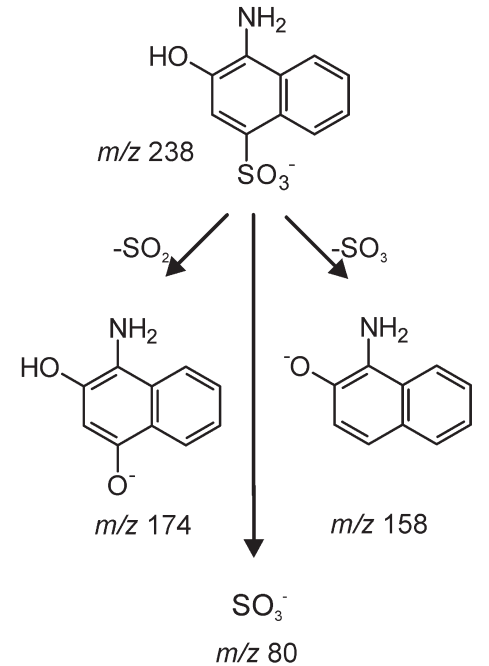

Fig. 1.15 ESI-MS spectra and suggested fragmentation schemes for the molecular ions of 1-amino-2-hydroxy-naphthalene-4-sulfonic acid in negative (left side) and positive (right side) ion mode. In negative ion mode a sulfo- $(\mathrm{m} / \mathrm{z})$<smiles>CC(=O)N(C)[C@@H](C)N</smiles><smiles></smiles><smiles>[R16]c1cccc2c(O)cc(C(C)O)cc12</smiles>

nate anion is generated first and further fragmentations occur at this group. In positive ion mode an ammonium cation is generated, leading to fragments with the positive charge located at the aromatic system.

$m / z 240 \rightarrow 159$ in the positive ion mode (Fig. 1.15). Thus, not only the intensity of the molecular ion signal, but also the intensity and the selectivity of transitions selected for multiple reaction monitoring are important criteria to be considered in method development and may influence the decision on the mode of ionization.

\subsubsection{Mass Spectrometers and Modes of Operation}

\section{Triple-Quadrupole and Ion-Trap Mass Spectrometers}

The development of mass spectrometer instrumentation has continued steadily. Single-stage quadrupole MS is no longer considered adequate for the detection of 
trace pollutants from environmental samples. For routine determination of trace compounds, these instruments are replaced by triple-stage quadrupole mass spectrometers (QqQ-MS) and ion trap mass spectrometers. For quantitative analysis of target analytes, triple quad MS operated in multiple-reaction monitoring mode provides unsurpassed sensitivity and a large dynamic range of several orders of magnitude.

Meanwhile, new mass spectrometers are commercially available that are using a so-called linear ion trap coupled behind a quadrupole. These instruments can be used for high-sensitivity MRM determination of target analytes when the second mass spectrometer is operated as a quadrupole and also for high-sensitivity product ion spectra recording when the second mass spectrometer is operated as an ion trap.

Recent years have seen an increasing awareness of quality control in environmental analysis also. This development was fostered by a directive concerning quality control in residue analysis released by the Commission of the European Union [208]. This directive proposes a system of identification points and requires a minimum of three such points to confirm a positive finding. When two precursor-product ion pairs are recorded, their relative intensity is compared with that of a reference standard, and if the chromatographic retention times coincide within certain limits these criteria are fulfilled. Difficulties arise, however, when an analyte exhibits only one intense product ion and no second transition is available for confirmation.

The limited mass resolution of quadrupole- and ion-trap mass spectrometers limits their use for the identification of unknown compounds, as it does not allow the determination of elemental compositions under routine conditions. Triplequadrupole mass spectrometers can be used to screen for compounds with a common functional group or a common structural element by using either neutral loss or precursor ion scans. By these approaches, metabolites of a known parent compound can be determined, provided that the transformation products retain the substructure or functional group which the detection was based on [209]. However, the sensitivity of detection drastically decreases in the parent ion scan, in which the first mass analyzer is scanning and, even more so, in neutral loss modes when both mass analyzers are operated in scanning mode [132].

\section{Time-of-Flight MS}

Time-of-flight mass spectrometers (TOF-MS) and quadrupole-time-of-flight mass spectrometers (Q-TOF-MS) offer a mass resolution of 5000 to $>15000$ (fwhm). For small molecules, this is often sufficient to determine the molecular formulas of unknowns (TOF-MS) and of their fragment ions (Q-TOF-MS).

Despite its inherent high sensitivity for whole-spectrum recording, TOF-MS cannot yet compete with triple-quadrupole-MS operated in MRM mode in both sensitivity of detection and dynamic range ( 2 orders of magnitude distance). Therefore, the main field of TOF and Q-TOF MS is qualitative analysis rather than the quantitation of known target analytes. Typical applications are metabolite identification, verification of positive findings, and screening for unknowns. Q-TOF- 
MS is much more useful than TOF-MS in environmental application, because product ion spectra can be detected with high mass resolution, which is essential for the identification of unknowns [210]. In this way, not only can the elemental formula of a molecular ion be determined, but unambiguous information on some of its structural characteristics can be obtained. Conversely, exact mass information on fragment ions of an unknown molecular ion supports its molecular formula determination: (a) the exact mass difference between the product and the precursor is an additional piece of information, and (b) often only one possible combination of the proposed elemental compositions of fragment ions and the molecular ion is consistent (Table 1.3).

TOF-MS has been used to confirm the occurrence of herbicides [211] or to identify previously unknown contaminants in environmental samples [212] and industrial wastewater [213]. Q-TOF-MS is also useful to elucidate fragmentation processes of polar pollutants [214]. Because of its high resolution and good sensitivity over a full mass range, TOF-MS would seem to be advantageous for screening purposes. The use of a Q-TOF can be even more useful, as it enables product ion spectra to be recorded for further identification [210]. The high mass resolution provided by a TOF-MS can be used to reduce the chemical noise in selected ion chromatograms [212]. Though this is an illustrative application of the high resolving power of these instruments, the practical use of such chromatograms is limited.

A second advantage, besides whole-spectra recording with high sensitivity, is the recording speed of TOF-MS, which is in the range of nanoseconds for one whole spectrum. This high speed is due to the fact that no scanning is required as in

Tab. 1.3 Exact masses of the molecular anion and one fragment ion of ethylhexyl phosphate (MEHP) identified by LC-Q-TOF-MS in a municipal wastewater. Based on the determined exact masses, possible elemental compositions were calculated for the two anions and for their mass difference. The only consistent combination of elemental formulas for the three masses is highlighted in bold and corresponds to MEHP. Data from [53].

\begin{tabular}{|c|c|c|c|c|}
\hline Anion & $\begin{array}{l}\text { Measured } \\
(\mathrm{m} / \mathrm{z})\end{array}$ & $\begin{array}{l}\text { Proposed } \\
\text { formulas }^{[a]}\end{array}$ & $\begin{array}{l}\text { Calculated } \\
(m / z)\end{array}$ & $\begin{array}{l}\text { Error } \\
(m D a)\end{array}$ \\
\hline Molecular & 209.0938 & $\begin{array}{l}\mathrm{C}_{8} \mathrm{H}_{18} \mathrm{O}_{4} \mathrm{P} \\
\mathrm{C}_{6} \mathrm{H}_{16} \mathrm{~N}_{3} \mathrm{O}_{3} \mathrm{P} \\
\mathrm{C}_{9} \mathrm{H}_{17} \mathrm{~N}_{5} \mathrm{O}_{2} \\
\mathrm{C}_{11} \mathrm{H}_{16} \mathrm{NOP} \\
\mathrm{C}_{7} \mathrm{H}_{19} \mathrm{~N}_{2} \mathrm{O}_{1} \mathrm{P}_{2}\end{array}$ & $\begin{array}{l}209.0943 \\
209.0929 \\
209.0959 \\
209.0970 \\
209.0973\end{array}$ & $\begin{array}{r}-0.5 \\
0.9 \\
-2.1 \\
-3.2 \\
-3.5\end{array}$ \\
\hline Product & 78.9623 & $\begin{array}{l}\mathrm{H}_{2} \mathrm{NPS} \\
\mathrm{PO}_{3}\end{array}$ & $\begin{array}{l}78.9646 \\
78.9585\end{array}$ & $\begin{array}{r}-2.3 \\
3.8\end{array}$ \\
\hline Difference & 130.1315 & $\begin{array}{l}\mathrm{C}_{16} \mathrm{H}_{16} \mathrm{~N}_{3} \\
\mathrm{C}_{8} \mathrm{H}_{18} \mathrm{O}\end{array}$ & $\begin{array}{l}130.1344 \\
130.1358\end{array}$ & $\begin{array}{l}-2.9 \\
-4.3\end{array}$ \\
\hline
\end{tabular}

\footnotetext{
a formulas proposed using the following conditions: mass tolerance of $\pm 5 \mathrm{mDa}$.

Element composition: $\mathrm{C}=0-20, \mathrm{H}=0-40, \mathrm{~N}=0-5, \mathrm{O}=0-6, \mathrm{~S}=0-1$, and $\mathrm{P}=0-2$.
} 
quadrupole or ion-trap mass spectrometers. This characteristic makes TOF detectors ideal for very fast chromatographic separations that result in narrow chromatographic peaks, as in ultraperformance liquid chromatography (UPLC) [215], where peak capacities of several hundreds are achievable [216].

\subsubsection{Quantitation Strategies and Matrix Effects}

The high selectivity and low chemical noise usually experienced when using LCAPI-MS in MRM-mode provides unsurpassed detection limits in the analysis of polar pollutants. However, this selectivity weakens our awareness that the target analyte is only an extreme minority of the whole amount of sample injected onto the analytical column. This so-called matrix may affect the ionization of the analytes of interest and may result in an erroneous quantitation by LC-MS. A typical problem is coextracted humic material in the trace analysis of acidic pesticides from ground- and surface waters [147, 173, 217-219]. But in general, any coeluting organic compound can interfere with the ionization of a target analyte [220-222]. In quantitative analysis from sampling campaigns in the field, matrix effects may vary considerably from sample to sample and from sampling to sampling [147, 217]. As the sample matrix is also subjected to chromatographic separation, it may vary for each analyte in a multi-component analysis during its time of entry into the interface, as do the matrix effects [223]. Therefore, matrix effects are, indeed, often the major hurdle in the routine analysis of environmental samples by LCMS.

To improve this situation, the highest priority should be given to any approach that reduces the matrix effects, either by more selective extraction or improved chromatography. More selective extraction may be achievable by two-step extraction at different $\mathrm{pH}$-values [219]. For very polar analytes, a precleaning step by $\mathrm{C}_{18^{-}}$ SPE can be used to remove less polar matrix constituents prior to analyte extraction with a polymeric sorbent $[10,190]$. A triple-phase liquid-phase microextraction has recently proven useful for this purpose [94]. Even a clean-up of extracts before the LC-MS analysis may be used (SEC, [205]), although this introduces new sources of error. However, environmental analysis often aims at determining a larger number of analytes covering a broad range of physico-chemical properties. This substantially limits the perspectives of clean-up options, which rely on differences in the physico-chemical properties of the analytes and the sample matrix.

There may be two other options to reduce matrix effects. (A) It was recently pointed out, that signal suppression by coeluting matrix can be considerably reduced by directing lower flow volumes into the ESI source [206, 207]. Moreover, the increasing sensitivity of mass spectrometers provides increasing opportunities to omit analyte enrichment and to directly inject the aqueous sample into the LCMS system. It may be astonishing that both these strategies can reduce matrix effects even though the concentration ratio of the target analyte to the matrix components is not changed. However, in both cases less material is entering the ionization interface at a given time. Thus, competition for whatever may limit the ionization efficacy, electrical charge or droplet surface area, can be avoided. 


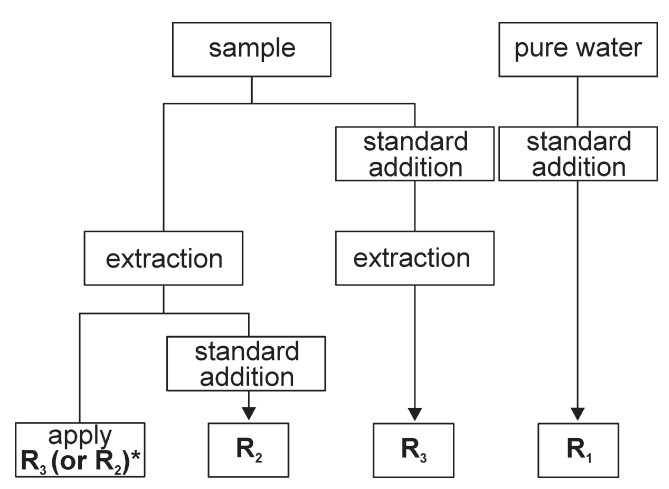

Fig. 1.16 Calibration scheme for the independent determination of matrix effects $\left(R_{2} / R_{1}\right)$ and SPE recovery $\left(R_{3} / R_{2}\right)$ in ESI-MS. * The response factor $R_{3}$ (or $R_{2}$, if extraction was shown to be quantitative) may finally be applied for quantitation of analytes in samples.

If matrix effects cannot be reduced, compensation is necessary to provide quantitatively accurate results. This can best be done by an isotopically labeled standard for each of the analytes included in one method. Unfortunately, such compounds are often not available for environmentally relevant analytes. Then, addition of a standard must be performed. In the frequent case that analytes are enriched prior to the LC-MS analysis, a low signal for a standard compound added to a sample prior to extraction as compared to a reference solution may result from incomplete extraction or from suppression of its signal due to matrix. Therefore, the recovery of the enrichment procedure and the matrix effects need to be distinguished. The clearest results are obtained by performing three calibrations (Fig. 1.16): one in pure aqueous solution $\left(R_{1}\right)$, one by standard addition to aliquots of a sample extract $\left(R_{2}\right)$, and one by standard addition to a sample before enrichment $\left(R_{3}\right)$. The ratio of the response factors $R_{2} / R_{1}$ describes matrix effects, whereas $R_{3} / R_{2}$ describes the recovery of the enrichment process.

If one is confident in having a uniform matrix within a series of samples, calibration can be performed by standard addition to only one sample of a series [220]. If the matrix varies from sample to sample, standard addition to each sample would have to be performed for each sample. This, however, leads to three to four times higher sample numbers, and, although it can correct for sensitivity losses by matrix compounds, this approach cannot avoid the loss of sensitivity.

\section{6}

\section{Conclusions}

Recent years have seen significant progress in analytical methods for polar pollutants. Polymeric sorbents have improved the extractability of polar pollutants from aqueous samples. GC-based analyses often require derivatization of polar analytes, namely when they are ionic, but still offer the highest chromatographic resolution. With regard to mass spectrometric determination, ESI-MS appears to surmount all previous limitations with respect to analyte polarity, and ion chromatography and ion-pair chromatography have similarly tranformed chromatographic separation. 
Instrumental development of mass spectrometers will continue to provide the analytical chemist with both increasing sensitivity and improved mass resolution. At the same time, the effort involved in analyzing polar pollutants in environmental samples steadily decreases while productivity increases. The development of multi-component methods across several chemical classes of compounds and the increasing use of direct injection of aqueous samples into LC-MS systems are the most recent examples of this trend.

Many of the results and much of the knowledge presented in the following chapters of this book could not have been gathered without the analytical methods for polar pollutants outlined in this chapter. Furthermore, environmental analytical chemistry provides the means to continue and extend the study of the occurrence and behavior of polar pollutants in all compartments of the water cycle.

\section{References}

1 M. Petrovic, D. Barcelo, Trends Anal. Chem. 2004, 23, 762-771.

2 T. P. Knepper, D. Barceló, P. de Voogt (Ed.), Analysis and fate of surfactants in the aquatic environment, Elsevier, Amsterdam, 2003.

3 M. J. M. Wells, L. Z. Yu, J. Chromatogr. A 2000, 885, 237-250.

4 Y. Picó, G. Font, J. C. Moltó, J. Mañes, J. Chromatogr. A 2000, 885, 251-271.

5 W. L. Budde, Mass Spectrom. Rev. 2004, 23, 1-24.

6 Y. Picó, C. Blasco, G. Font, Mass Spectrom. Rev. 2004, 23, 45-85.

7 R. B. Geerdink, W. M. A. Niessen, U. A. T. Brinkman, J. Chromatogr. A 2002, 970, 65-93.

8 V. Andreu, Y. Picó, Trends Anal. Chem. 2004, 23, 772-789.

9 M. Rompa, E. Kremer, B. Zygmunt, Anal. Bioanal. Chem. 2003, 377, 590-599.

10 T. A. Ternes, Trends Anal. Chem. 2001, 20, 419-434.

11 J. Beausse, Trends Anal. Chem. 2004, 23, 753-761.

12 M. S. Díaz-Cruz, M. J. López de Alda, D. Barceló, Trends Anal. Chem. 2003, 22, 340-351.

13 J. B. Quintana, J. Carpinteiro, I. Rodríguez in Analysis, fate and removal of pharmaceuticals in the water cycle (Ed. M. Petrovic, D. Barcelo), Elsevier, Amsterdam. In press.

14 S. D. Richardson, Trends Anal. Chem. 2003, 22, 666-684.
15 T. P. Knepper, Trends Anal. Chem. 2003, 22, 708-724.

16 M. Sillanpää, M. L. Sihvonen, Talanta 1997, 44, 1487-1497.

17 R. B. Lee, K. Sarafin, T. E. Peart, M. L. Svoboda, Water Qual. Res. J. Canada 2003, 38, 667-682.

18 S. Reddy, C. R. Iden, B. J. Brownawell, Anal. Chem. 2005, 77, 7032-7038.

19 M. Stumpf, T. A. Ternes, K. Haberer, P. Seel, W. Baumann, Vom Wasser 1996, 86, 291-303.

20 D. Barceló, Trends Anal. Chem. 2004, 23, 677-679.

21 R. M. Smith, J. Chromatogr. A 2003, 1000, 3-27.

22 J. R. Dean, S. L. Cresswell in Sampling and sample preparation for field and laboratory (Ed. J. Pawliszyn), Elsevier, Amsterdam, 2002, pp. 559-586.

23 A. M. Jacobsen, B. Halling-Sørensen, F. Ingerslev, S. H. Hansen, J. Chromatogr. A 2004, 1038, 157-170.

24 Y. Kawagoshi, I. Fukunaga, H. Itoh, J. Mater. Cycles Waste Manag. 1999, 1, 53-61.

25 H. De Geus, B. N. Zegers, H. Lingeman, U. A. T. Brinkman, Int. J. Environ. Anal. Chem. 1994, 56, 119-132.

26 H. Singer, S. Müller, C. Tixier, L. Pillonel, Environ. Sci. Technol. 2002, 36, 4998-5004.

27 R. U. Halden, D. H. Paull, Environ. Sci. Technol. 2004, 38, 4849-4855. 
28 R. U. Halden, D. H. Paull, Environ. Sci. Technol. 2005, 39, 1420-1426.

29 D. Löffler, T. A. Ternes, J. Chromatogr. A 2003, 1021, 133-144.

30 T. A. Ternes, M. Bonerz, N. Herrmann, D. Löffler, E. Keller, B. Bagó Lacida, A. C. Alder, J. Chromatogr. A 2005, 1067, 213-223.

31 M. Himmelsbach, W. Buchberger, H. Miesbauer, Int. J. Environ. Anal. Chem. 2003, 83, 481-486.

32 E. M. Golet, A. Strehler, A. C. Alder, W. Giger, Anal. Chem. 2002, 74, 5455-5462.

33 E. M. Golet, A. C. Alder, A. Hartmann, T. A. Ternes, W. Giger, Anal. Chem. 2001, 73, 3632-3638.

34 C. Crescenzi, A. Di Corcia, M. Nazzari, R. Samperi, Anal. Chem. 2000, 72, 3050-3055.

35 S. B. Hawthorne, A. Kubátová in Sampling and sample preparation for field and laboratory (Ed. J. Pawliszyn), Elsevier, Amsterdam, 2002, pp. 587-608.

36 S. B. Hawthorne, Y. Yang, D. J. Miller, Anal. Chem. 1994, 66, 2912-2920.

37 F. Bruno, R. Curini, A. Di Corcia, I. Fochi, M. Nazzari, R. Samperi, Environ. Sci. Technol. 2002, 36, 4156-4161.

38 F. M. Guo, Q. X. Li, J. P. AlcantaraLicudine, Anal. Chem. 1999, 71, 1309-1315.

39 S. Campbell, Q. X. Li, Anal. Chim. Acta 2001, 434, 283-289.

40 A. Caballo-López, M. D. Luque de Castro, Chromatographia 2003, 58, 257-262.

41 H. R. Buser, T. Poiger, M. D. Müller, Environ. Sci. Technol. 1998, 32, 3449-3456.

42 K. Bester, Water Res. 2003, 37, 3891-3896.

43 US-EPA, EPA Method 552.1 Determination of haloacetic acids and dalapon in drinking water by ion excahnge liquid-solid extraction and gas chromatography with electron capture detector, Cincinnati, 1992.

44 US-EPA, EPA Method 552.2 Determination of haloacetic acids and dalapon in drinking water by liquid-liquid extraction, derivatization and gas chromatography with electron capture detector, Cincinnati, 1995.

45 J. B. Quintana, I. Rodríguez, Anal. Bioanal. Chem. 2006, 384, 1447-1461.
46 B. Cancho, F. Ventura, M. Galceran, A. Diaz, S. Ricart, Water Res. 2000, 34, 3380-3390.

47 C. W. Huck, G. K. Bonn, J. Chromatogr. A 2000, 885, 51-72.

48 F. Sacher, F. T. Lange, H. J. Brauch, I. Blankenhorn, J. Chromatogr. A 2001, 938, 199-210.

49 M. Petrovic, M. D. Hernando, M. S. Diaz-Cruz, D. Barcelo, J. Chromatogr. A 2005, 1067, 1-14.

50 E. M. Thurman, M. S. Mills, Solid-Phase Extraction. Principles and Practice, John Wiley \& Sons, New York, 1998.

51 ISO 16588: 2002. Water quality Determination of six complexing agents Gas-chromatographic method, International Standards Organization, Geneva, 2002.

52 R. Geschke, M. Zehringer, Fresenius J. Anal. Chem. 1997, 357, 773-776.

53 J. B. Quintana, R. Rodil, T. Reemtsma, Anal. Chem. 2006, 78, 1644-1650.

54 J. B. Quintana, S. Weiss, T. Reemtsma, Water Res. 2005, 39, 2654-2664.

55 US-EPA, EPA Method 549.1 Determination of diquat and paraquat in drinking water by liquid-solid extraction and high performance liquid chromatography with ultraviolet detection, Cincinatti, 1992.

56 M. C. Carson, J. Chromatogr. A 2000, 885, 343-350.

57 E. Pocurull, M. Calull, R. M. Marcé, F. Borrull, J. Chromatogr. A 1996, 719, 105-112.

58 M. C. Hennion, J. Chromatogr. A 2000, 885, 73-95.

59 A. Putschew, S. Schittko, M. Jekel, J. Chromatogr. A 2001, 930, 127-134.

60 O. Núñez, E. Moyano, M. T. Galcerán, J. Chromatogr. A 2002, 946, 275-282.

61 N. Fontanals, M. Galia, R. M. Marce, F. Borrull, J. Chromatogr. A 2004, 1030, 63-68.

62 N. Fontanals, P. Puig, M. Galia, R. M. Marce, F. Borrull, J. Chromatogr. A 2004, 1035, 281-284.

63 N. Fontanals, R. M. Marcé, F. Borrull, Trends Anal. Chem. 2005, 24, 394-406.

64 I. Rodríguez Pereiro, R. González Irimia, E. Rubí Cano, R. Cela Torrijos, Anal. Chim. Acta 2004, 524, 249-256. 
65 C. Zwiener, T. Glauner, F. H. Frimmel, Anal. Bioanal. Chem. 2002, 372, 615-621.

66 S. Öllers, H. P. Singer, P. Fassler, S. R. Muller, J. Chromatogr. A 2001, 911, 225-234.

67 I. Rodríguez, J. B. Quintana, J. Carpinteiro, A. M. Carro, R. A. Lorenzo, R. Cela, J. Chromatogr. A 2003, 985, 265-274.

68 R. Rodil, J. B. Quintana, T. Reemtsma, Anal. Chem. 2005, 77, 3083-3089.

69 A. Kloepfer, M. Jekel, T. Reemtsma, J. Chromatogr. A 2004, 1058, 81-88.

70 S. Weiss, T. Reemtsma, Anal. Chem. 2005, 77, 7415-7420.

71 S. Weigel, R. Kallenborn, H. Hühnerfuss, J. Chromatogr. A 2004, 1023, 183-195.

72 L. Grey, B. Nguyen, P. Yang, J. Chromatogr. A 2002, 958, 25-33.

73 F. Hernández, J. V. Sancho, O. Pozo, A. Lara, E. Pitarch, J. Chromatogr. A 2001, 939, 1-11.

74 A. Asperger, J. Efer, T. Koal, W. Engewald, J. Chromatogr. A 2002, 960, 109-119.

75 A. C. Hogenboom, P. Speksnijder, R. J. Vreeken, W. M. A. Niessen, U. A. T. Brinkman, J. Chromatogr. A 1997, 777 , 81-90.

76 F. Chapuis, V. Pichon, M. C. Hennion, LC GC Eur. 2004, 17, 408-412, 414, 416-417.

77 E. Caro, R. M. Marcé, P. A. G. Cormack, D. C. Sherrington, F. Borrull, J. Chromatogr. A 2003, 995, 233-238.

78 E. Caro, M. Masqué, R. M. Marcé, F. Borrull, P. A. G. Cormack, D. C. Sherrington, J. Chromatogr. A 2002, 963, 169-178.

79 R. P. Belardi, J. B. Pawliszyn, Wat. Pollution Res. J. Canada 1989, 24, 179-191.

80 J. Pawliszyn in Sampling and sample preparation for field and laboratory (Ed. J. Pawliszyn), Elsevier, Amsterdam, 2002, pp. 389-477.

81 J. Pawliszyn, Solid phase microextraction: theory and practice, Wiley-VCH, New York, 1997.

82 H. Kataoka, Anal. Bioanal. Chem. 2002, 373, 31-45.
83 M. Moeder, S. Schrader, M. Winkler, P. Popp, J. Chromatogr. A 2000, 873, 95-106.

84 E. E. Stashenko, J. R. Martínez, Trends Anal. Chem. 2004, 23, 553-561.

85 J. P. Lamas, C. Salgado-Petinal, C. García-Jares, M. Llompart, R. Cela, M. Gómez, J. Chromatogr. A 2004, 1046, 241-247.

86 T. Zimmermann, W. J. Ensinger, T. C. Schmidt, Anal. Chem. 2004, 76, 1028-1038.

87 P. Canosa, I. Rodríguez, E. Rubi, R. Cela, J. Chromatogr. A 2005, 1072, 107-115.

88 I. Rodríguez, J. Carpinteiro, J. B. Quintana, A. M. Carro, R. A. Lorenzo, R. Cela, J. Chromatogr. A 2004, 1024, 1-8.

89 I. Rodríguez, E. Rubí, R. González, J. B. Quintana, R. Cela, Anal. Chim. Acta 2005, 537, 259-266.

90 E. Psillakis, N. Kalogerakis, Trends Anal. Chem. 2002, 21, 53-63.

91 K. E. Rasmussen, S. Pedersen-Bjergaard, Trends Anal. Chem. 2004, 23, 1-10.

92 E. Psillakis, N. Kalogerakis, Trends Anal. Chem. 2003, 22, 565-574.

93 J. A. Jönsson, L. Mathiasson, LC GC Eur. 2003, 16, 683-690.

94 J. B. Quintana, R. Rodil, T. Reemtsma, J. Chromatogr. A 2004, 1061, 19-26.

95 J. Segura, R. Ventura, C. Jurado, J. Chromatogr. B 1998, 713, 61-90.

96 R. J. Wells, J. Chromatogr. A 1999, 843, $1-18$.

97 J. L. Little, J. Chromatogr. A 1999, 844, $1-22$.

98 J. M. Rosenfeld in Sampling and sample preparation for field and laboratory (Ed. J. Pawliszyn), Elsevier, Amsterdam, 2002, pp. 609-668.

99 K. Blau, J. M. Halket, Handbook of derivatives for chromatography, John Wiley \& Sons, Chichester, 1993.

$100 \mathrm{~J}$. Drozd, Chemical derivatization in gas chromatography, Elsevier, Amsterdam, 1981.

101 H. R. Buser, M. D. Müller, N. Theobald, Environ. Sci. Technol. 1998, 32, 188-192.

102 H. R. Buser, T. Poiger, M. D. Müller, Environ. Sci. Technol. 1999, 33, 2529-2535.

103 H. M. Kuch, K. Ballschmiter, Environ. Sci. Technol. 2001, 35, 3201-3206. 
104 A. Zapf, H. J. Stan, J. High Resolut. Chromatogr. 1999, 22, 83-88.

105 R. Alzaga, A. Peña, L. Ortiz, J. M. Bayona, J. Chromatogr. A 2003, 999, 51-60.

106 R. Alzaga, J. M. Bayona, J. Chromatogr. A 2004, 1042, 155-162.

107 C. Zwiener, F. H. Frimmel, Water Res. 2000, 34, 1881-1885.

108 C. Zwiener, T. Glauner, F. H. Frimmel, J. High Resolut. Chromatogr. 2000, 23, 474-478.

109 C. Zwiener, S. Seeger, T. Glauner, F. H. Frimmel, Anal. Bioanal. Chem. 2002, 372, 569-575.

110 T. Henriksen, B. Svensmark, B. Lindhardt, R. K. Juhler, Chemosphere 2001, 44, 1531-1539.

111 M. Ibáñez, O. J. Pozo, J. V. Sancho, F. J. Lopez, F. Hernández, J. Chromatogr. A 2005, 1081, 145-155.

112 M. Llompart, M. Lourido, P. Landín, C. García-Jares, R. Cela, J. Chromatogr. A 2002, 963, 137-148.

113 I. Rodríguez, M. I. Turnes, M. C. Mejuto, R. Cela, J. Chromatogr. A 1996, 721, 297-304.

114 D. Li, J. Park, J. R. Oh, Anal. Chem. 2001, 73, 3089-3095.

115 G. R. Boyd, H. Reemtsma, D. A. Grimm, S. Mitra, Sci. Total Environ. 2003, 311, 135-149.

116 T. Heberer, H. J. Stan, Anal. Chim. Acta 1997, 341, 21-34.

117 S. Zühlke, U. Dünnbier, T. Heberer, J. Chromatogr. A 2004, 1050, 201-209.

118 K. Reddersen, T. Heberer, J. Sep. Sci. 2003, 26, 1443-1450.

119 J. B. Quintana, J. Carpinteiro,

I. Rodriguez, R. A. Lorenzo, A. M. Carro, R. Cela, J. Chromatogr. A 2004, 1024, 177-185.

120 H. R. Buser, M. D. Müller, Environ. Sci. Technol. 1998, 32, 626-633.

121 H. R. Buser, M. D. Müller, T. Poiger, M. E. Balmer, Environ. Sci. Technol. 2002, 36, 221-226.

122 E. Matisová, M. Dömötörová, J. Chromatogr. A 2003, 1000, 199-221.

123 M. Hada, M. Takino, T. Yamagami, S. Daishima, K. Yamaguchi, J. Chromatogr. A 2000, 874, 81-90.
124 C. Bicchi, C. Brunelli, C. Cordero, P. Rubiolo, M. Galli, A. Sironi, J. Chromatogr. A 2005, 1071, 3-12.

125 J. Dalluge, J. Beens, U. A. T. Brinkman, J. Chromatogr. A 2003, 1000, 69-108.

126 P. Marriott, R. Shellie, Trends Anal. Chem. 2002, 21, 573-583.

127 T. Ieda, Y. Horii, G. Petrick, N. Yamashita, N. Ochiai, K. Kannan, Environ. Sci. Technol. 2005, 39, 7202-7207.

128 M. Stumpf, T. A. Ternes, K. Haberer, W. Baumann, Vom Wasser 1998, 91, 291-303.

129 P. M. Hoai, S. Tsunoi, M. Ike, Y. Kuratani, K. Kudou, P. H. Viet, M. Fujita, M. Tanaka, J. Chromatogr. A 2003, 1020, 161-171.

130 R. Bossi, K. V. Vejrup, B. B. Mogensen, W. A. H. Asman, J. Chromatogr. A 2002, 957, 27-36.

131 J. Slobodnik, A. C. Hogenboom, J. J. Vreuls, J. A. Rontree, B. L. M. van Baar, W. M. A. Niessen, U. A. T. Brinkman, J. Chromatogr. A 1996, 741, 59-74.

132 T. Reemtsma, J. Chromatogr. A 2003, 1000, 477-501.

133 T. Reemtsma, Trends Anal. Chem. 2001, 20, 533-542.

134 T. Reemtsma, Trends Anal. Chem. 2001, 20, 500-517.

135 C. Zwiener, F. H. Frimmel, Anal. Bioanal. Chem. 2004, 378, 851-861.

136 M. J. F. Suter, S. Riediker, W. Giger, Anal. Chem. 1999, 71, 897-904.

137 M. C. Alonso, M. Castillo, D. Barcelo, Anal. Chem. 1999, 71, 2586-2593.

138 T. Storm, T. Reemtsma, M. Jekel, J. Chromatogr. A 1999, 854, 175-185.

139 T. Storm, C. Hartig, T. Reemtsma, M. Jekel, Anal. Chem. 2001, 73, 589-595.

140 J. E. B. McCallum, S. A. Madison, S. Alkan, R. L. Depinto, R. U. R. Wahl, Environ. Sci. Technol. 2000, 34, 5157-5164.

141 T. Reemtsma, J. Chromatogr. A 2001, 919, 289-297.

142 A. Di Corcia, F. Casassa, C. Crescenzi, A. Marcomini, R. Samperi, Environ. Sci. Technol. 1999, 33, 4112-4118.

143 E. Gonzalez Mazo, M. Honing, D. Barcelo, A. GomezParra, Environ. Sci. Technol. 1997, 31, 504-510. 
144 P. Eichhorn, T. P. Knepper, Environ. Toxicol. Chem. 2002, 21, 1-8.

145 J. Riu, E. Gonzalez-Mazo, A. GomezParra, D. Barcelo, Chromatographia 1999, 50, 275-281.

146 P. Eichhorn, M. E. Flavier, M. L. Paje, T. P. Knepper, Sci. Total Environ. 2001, 269, 75-85.

147 W. M. A. Niessen, J. Chromatogr. A 1998 , 794, 407-435.

148 R. Loos, D. Barcelo, J. Chromatogr. A 2001, 938, 45-55.

149 K. H. Bauer, T. P. Knepper, A. Maes, V. Schatz, M. Voihsel, J. Chromatogr. A 1999, 837, 117-128.

150 T. P. Knepper, A. Werner, G. Bogenschütz, J. Chromatogr. A 2005 , 1085, 240-246.

151 R. N. Collins, B. C. Onisko, M. J. McLaughlin, G. Merrington, Environ. Sci. Technol. 2001, 35, 2589-2593.

152 J. A. Ballantine, D. E. Games, P. S. Slater, Rapid Commun. Mass Spectrom. 1995, 9, 1403-1410.

153 R. A. Yokley, L. C. Mayer, S. B. Huang, J. D. Vargo, Anal. Chem. 2002, 74, 3754-3759.

154 I. Ferrer, E. M. Thurman, D. Barcelo, Anal. Chem. 1997, 69, 4547-4553.

155 L. H. Levine, J. L. Garland, J. V. Johnson, Anal. Chem. 2002, 74, 2064-2071.

156 G. Ohlenbusch, C. Zwiener, R. U. Meckenstock, F. H. Frimmel, J. Chromatogr. A 2002, 967, 201-207.

157 X. S. Miao, B. G. Koenig, C. D. Metcalfe, J. Chromatogr. A 2002, 952, 139-147.

158 T. A. Ternes, R. Hirsch, Environ. Sci. Technol. 2000, 34, 2741-2748.

159 T. Sasaki, T. Iida, T. Nambara, J. Chromatogr. A 2000, 888, 93-102.

160 R. Hirsch, T. A. Ternes, A. Lindart, K. Haberer, R. D. Wilken, Fresenius J. Anal. Chem. 2000, 366, 835-841.

161 J. B. Quintana, T. Reemtsma, Rapid Commun. Mass Spectrom. 2004, 18, 765-774.

162 S. K. Johnson, L. L. Houk, D. C. Johnson, R. S. Houk, Anal. Chim. Acta 1999, 389, 1-8.

163 W. Ahrer, W. Buchberger, J. Chromatogr. A 1999, 854, 275-287.

164 R. Castro, E. Moyano, M. T. Galceran, J. Chromatogr. A 2001, 914, 111-121.
165 R. Castro, E. Moyano, M. T. Galceran, J. Chromatogr. A 1999, 830, 145-154.

166 M. Takino, S. Daishima, K. Yamaguchi, J. Chromatogr. A 1999, 862, 191-197.

167 S. A. Gustavsson, J. Samskog, K. E. Markides, B. Langstrom, J. Chromatogr. A 2001, 937, 41-47.

168 I. Ferrer, E. T. Furlong, Environ. Sci. Technol. 2001, 35, 2583-2588.

169 M. J. Ford, L. W. Tetler, J. White, D. Rimmer, J. Chromatogr. A 2002, 952, 165-172.

170 N. Wang, W. L. Budde, Anal. Chem. 2001, 73, 997-1006

171 T. Reemtsma, Rapid Commun. Mass Spectrom. 2000, 14, 1612-1618.

172 X. S. Miao, C. D. Metcalfe, Anal. Chem. 2003, 75, 3731-3738.

173 E. T. Furlong, M. R. Burkhardt, P. M Gates, S. L. Werner, W. A. Battaglin, Sci. Total Environ. 2000, 248, 135-146.

174 A. Cohen, K. Klint, S. Bowadt, P. Persson, J. A. Jonsson, J. Chromatogr. A 2001, 927, 103-110.

175 P. L. Ferguson, C. R. Iden, B. J. Brownawell, Anal. Chem. 2000, 72, 4322-4330.

176 M. Petrovic, A. Diaz, F. Ventura, D. Barcelo, Anal. Chem. 2001, 73, 5886-5895.

177 P. L. Ferguson, C. R. Iden, B. J. Brownawell, J. Chromatogr. A 2001, 938, 79-91.

178 C. Crescenzi, A. Dicorcia, R. Samperi, A. Marcomini, Anal. Chem. 1995, 67, 1797-1804.

179 K. A. Krogh, K. V. Vejrup, B. B. Mogensen, B. Halling-Sorensen, J. Chromatogr. A 2002, 957, 45-57.

180 G. Cretier, C. Podevin, J. L. Rocca, Analysis 1999, 27, 758-764.

181 P. Eichhorn, T. P. Knepper, J. Chromatogr. A 1999, 854, 221-232.

182 P. Billian, W. Hock, R. Doetzer, H. J. Stan, W. Dreher, Anal. Chem. 2000, 72, 4973-4978.

183 T. Ternes, M. Bonerz, T. Schmidt, J. Chromatogr. A 2001, 938, 175-185.

184 X. S. Miao, F. Bishay, M. Chen, C. D. Metcalfe, Environ. Sci. Technol. 2004, 38, 3533-3541.

185 P. Eichhorn, T. P. Knepper, J. Mass Spectrom. 2001, 36, 677-684. 
186 F. Bruno, R. Curini, A. Di Corcia, M. Nazzari, R. Samperi, Rapid Commun. Mass Spectrom. 2001, 15, 1391-1400.

187 S. W. Yang, J. Cha, K. Carlson, Rapid Commun. Mass Spectrom. 2004, 18, 2131-2145.

188 G. Hamscher, S. Sczesny, H. Hoper, H. Nau, Anal. Chem. 2002, 74, 1509-1518.

189 M. E. Lindsey, M. Meyer, E. M. Thurman, Anal. Chem. 2001, 73, 4640-4646.

190 C. Hartig, T. Storm, M. Jekel, J. Chromatogr. A 1999, 854, 163-173.

191 R. J. Vreeken, P. Speksnijder, I. Bobeldijk-Pastorova, T. H. M. Noij, J. Chromatogr. A 1998, 794, 187-199.

192 L. Goodwin, J. R. Startin, B. J. Keely, D. M. Goodall, J. Chromatogr. A 2003, 1004, 107-119.

193 A. Di Corcia, M. Nazzari, R. Rao, R. Samperi, E. Sebastiani, J. Chromatogr. A 2000, 878, 87-98.

194 J. Klein, L. Alder, J. AOAC Int. 2003, 86, 1015-1037.

195 B. J. Vanderford, R. A. Pearson, D. J. Rexing, S. A. Snyder, Anal. Chem. 2003, 75, 6265-6274.

196 A. L. Batt, D. S. Aga, Anal. Chem. 2005, 77, 2940-2947.

197 B. Kafkova, Z. Bosakova, E. Tesarova, P. Coufal, J. Chromatogr. A 2005, 1088, 82-93.

198 G. D’Orazio, Z. Aturki, M. Cristalli, M. G. Quaglia, S. Fanali, J. Chromatogr. A 2005, 1081, 105-113.

199 P. S. Bonato, M. Del Lama, R. de Carvalho, J. Chromatogr. B 2003, 796, 413-420.

200 K. Otsuka, C. J. Smith, J. Grainger, J. R. Barr, D. G. Patterson, N. Tanaka, S. Terabe, J. Chromatogr. A 1998, 817, 75-81.

201 E. M. Thurman, I. Ferrer, D. Barcelo, Anal. Chem. 2001, 73, 5441-5449.

202 S. Zuehlke, U. Duennbier, T. Heberer, Anal. Chem. 2004, 76, 6548-6554.

203 R. King, R. Bonfiglio, C. FernandezMetzler, C. Miller-Stein, T. Olah, J. Am. Soc. Mass Spectrom. 2000, 11, 942-950.

204 B. K. Matuszewski, M. L. Constanzer, C. M. Chavez-Eng, Anal. Chem. 1998, 70, 882-889.
205 M. P. Schlüsener, K. Bester, Rapid Commun. Mass Spectrom. 2005, 19, 3269-3278.

206 E. T. Gangl, M. Annan, N. Spooner, P. Vouros, Anal. Chem. 2001, 73, 5635-5644.

207 A. Kloepfer, J. B. Quintana, T. Reemtsma, J. Chromatogr. A 2005, 1067, 153-160.

208 Commission Decision (2002/657/EC): Implementing Council directive 96/23/EC concerning the performance of analytical methods and the interpretation of results., Official J. Eur. Communities 2002, L067, 8-36.

209 R. Steen, I. Bobeldijk, U. A. T. Brinkman, J. Chromatogr. A 2001, 915 , 129-137.

210 I. Bobeldijk, J. P. C. Vissers, G. Kearney, H. Major, J. A. van Leerdam, J. Chromatogr. A 2001, 929, 63-74.

211 M. Maizels, W. L. Budde, Anal. Chem. 2001, 73, 5436-5440.

212 A. C. Hogenboom, W. M. A. Niessen, D. Little, U. A. T. Brinkman, Rapid Commun. Mass Spectrom. 1999, 13, 125-133.

213 T. P. Knepper, J. Chromatogr. A 2002, 974, 111-121.

214 K. Klagkou, F. Pullen, M. Harrison, A. Organ, A. Firth, G. J. Langley, Rapid Commun. Mass Spectrom. 2003, 17, 2373-2379.

215 R. Plumb, J. Castro-Perez, J. Granger, I. Beattie, K. Joncour, A. Wright, Rapid Commun. Mass Spectrom. 2004, 18, 2331-2337.

216 S. A. C. Wren, J. Pharm. Biomed. Anal. 2005, 38, 337-343.

217 R. Steen, A. C. Hogenboom, P. E. G. Leonards, R. A. L. Peerboom, W. P. Cofino, U. A. T. Brinkman, J. Chromatogr. A 1999, 857, 157-166.

218 E. Dijkman, D. Mooibroek, R. Hoogerbrugge, E. Hogendoorn, J. V. Sancho, O. Pozo, F. Hernandez, J. Chromatogr. A 2001, 926, 113-125.

219 R. B. Geerdink, A. Attema, W. M. A. Niessen, U. A. T. Brinkman, LC GC Int. 1998, 11, 361-372.

220 J. Zrostlikova, J. Hajslova, J. Poustka, P. Begany, J. Chromatogr. A 2002, 973 , 13-26. 
221 S. Ito, K. Tsukada, J. Chromatogr. A 2002, 943, 39-46.

222 R. Pascoe, J. P. Foley, A. I. Gusev, Anal. Chem. 2001, 73, 6014-6023.
223 B. K. Choi, D. M. Hercules, A. I. Gusev, Fresenius J. Anal. Chem. 2001, 369, 370-377. 